OPEN ACCESS

Edited by:

Ashok Hegde,

Georgia College and State University,

United States

Reviewed by:

Xiong Jiang,

Georgetown University, United States

Veena A. Nair,

University of Wisconsin-Madison,

United States

*Correspondence:

Mónica Emch

monica.emch@tum.de

Received: 09 January 2019 Accepted: 17 May 2019

Published: 12 June 2019

Citation:

Emch M, von Bastian CC and Koch K (2019) Neural Correlates of Verbal Working Memory: An fMRI Meta-Analysis.

Front. Hum. Neurosci. 13:180 doi: 10.3389/fnhum.2019.00180

\section{Neural Correlates of Verbal Working Memory: An fMRI Meta-Analysis}

\author{
Mónica Emch ${ }^{1,2,3 *}$, Claudia C. von Bastian ${ }^{4}$ and Kathrin Koch ${ }^{1,2,3}$ \\ ${ }^{1}$ Department of Neuroradiology, School of Medicine, Klinikum Rechts der Isar, Technical University of Munich, Munich, \\ Germany, ${ }^{2}$ TUM-Neuroimaging Center (TUM-NIC), Technical University of Munich, Munich, Germany, ${ }^{3}$ Graduate School of \\ Systemic Neurosciences, Ludwig-Maximilians-Universität, Martinsried, Germany, ${ }^{4}$ Department of Psychology, University of \\ Sheffield, Sheffield, United Kingdom
}

Verbal Working memory (VWM) capacity measures the ability to maintain and manipulate verbal information for a short period of time. The specific neural correlates of this construct are still a matter of debate. The aim of this study was to conduct a coordinate-based meta-analysis of $42 \mathrm{fMRI}$ studies on visual VWM in healthy subjects $(n=795$, males $=459$, females $=325$, unknown $=11$; age range: $18-75)$. The studies were obtained after an exhaustive literature search on PubMed, Scopus, Web of Science, and Brainmap database. We analyzed regional activation differences during $\mathrm{fMRI}$ tasks with the anisotropic effect-size version of seed-based d mapping software (ES-SDM). The results were further validated by performing jackknife sensitivity analyses and heterogeneity analyses. We investigated the effect of numerous relevant influencing factors by fitting corresponding linear regression models. We isolated consistent activation in a network containing fronto-parietal areas, right cerebellum, and basal ganglia structures. Regarding lateralization, the results pointed toward a bilateral frontal activation, a left-lateralization of parietal regions and a right-lateralization of the cerebellum, indicating that the left-hemisphere concept of vWM should be reconsidered. We also isolated activation in regions important for response inhibition, emphasizing the role of attentional control in vWM. Moreover, we found a significant influence of mean reaction time, load, and age on activation associated with vWM. Activation in left medial frontal gyrus, left precentral gyrus, and left precentral gyrus turned out to be positively associated with mean reaction time whereas load was associated with activation across the PFC, fusiform gyrus, parietal cortex, and parts of the cerebellum. In the latter case activation was mainly detectable in both hemispheres whereas the influence of age became manifest predominantly in the left hemisphere. This led us to conclude that future VWM studies should take these factors into consideration.

Keywords: verbal working memory, meta-analysis, neuroimaging, fMRI, subcortical areas, fronto-parietal activation, right cerebellum

\section{INTRODUCTION}

Working memory (WM) is a cognitive system that holds information available that is needed for complex cognition in the present moment (Baddeley, 2010; Oberauer and Hein, 2012). It has been shown that WM capacity is a strong predictor of a wide range of complex cognitive tasks such as analytic problem solving, language acquisition, and reading comprehension 
(Daneman and Carpenter, 1980; Engle et al., 1999; Wiley and Jarosz, 2012). There have been several attempts to understand the organization of human WM. The arguably most influential model is the multiple-component model proposed by Baddeley and Hitch (1974). The authors hypothesized the existence of a "central executive" component, which controls the incoming information and passes the information to two subsystems: the "phonological loop" and the "visuospatial sketchpad." Within the phonological loop, due to the interplay of its two componentsthe phonological store and the articulatory loop-the verbal material representation can be kept in an active state. Verbal information is processed in perceptual systems before it enters the phonological loop in which it is temporarily stored in the phonological store and maintained through the articulatory loop using subvocal rehearsal of the information. In addition to subvocal rehearsal, the articulatory loop is also thought to be involved whenever verbal information is presented visually: whereas auditory verbal information (e.g., spoken words) can directly enter the phonological store, visually presented verbal information (e.g., written words) must first be recoded into phonological information. In other words, subvocalization is necessary in order to reroute visually derived verbal material into the phonological store (Buchsbaum and D'Esposito, 2008). The visuospatial sketchpad is responsible for integrating visual and spatial information. Later, the "episodic buffer" was added (Baddeley, 2000). It binds the information from the different subsystems into integrated episodes. Alternative models proposed that WM holds any type of information in a state of heightened availability (Oberauer, 2010; Cowan et al., 2012) whereas others models have emphasized on the role of attentional control in WM (e.g., Kane and Engle, 2003; Unsworth and Engle, 2007). These different theoretical conceptualizations of WM are not necessarily mutually exclusive (Cowan et al., 2012), with common features including a variety of processes such as encoding, maintaining and retrieving information of various domains (e.g., letters, geometric forms, or words), and some attentional control mechanism that supports dealing with interference from irrelevant or distracting information. Thus, the neural correlates of WM may vary depending on the processes, the type of information, and the modality of stimulation (auditory or visual). Given the variety across studies with regard to WM domain and the lack of process differentiation in most studies, the present meta-analysis focused exclusively on visually presented verbal working memory (vWM) across all processes involved in WM.

\section{Visual Verbal Working Memory}

Several fMRI studies over the past years have specifically investigated the brain areas involved in vWM (Honey et al., 2000; Veltman et al., 2003; Chen and Desmond, 2005; Narayanan et al., 2005; Wolf et al., 2006). They basically corroborated the general notion that a variety of brain networks are activated during vWM mainly including areas in the prefrontal cortex (PFC) and the parietal cortex as well as cerebellar and basal ganglia regions (Paulesu et al., 1993; Petrides et al., 1993; Desmond et al., 1997; Crosson et al., 1999; Lewis et al., 2004; Chang et al., 2007; Buchsbaum et al., 2011; Thürling et al., 2012; Moore et al.,
2013; Chai et al., 2018). Previous meta-analyses have indicated that the left PFC might be predominantly involved in $\mathrm{vWM}$ processes whereas the right PFC seems to be more strongly involved in spatial WM, leading to a lateralization of this region due to different input (Wager and Smith, 2003; Owen et al., 2005). However, there is no general consensus on the functional organization of the PFC (Eriksson et al., 2015). Functional neuroimaging studies suggested that the articulatory loop is associated with the left inferior frontal cortex-where Broca's area is located -, left supplementary motor area (SMA), left premotor cortex (BA6), and left insula. The phonological store has been shown to be associated with the left BA 40, corresponding to the left supramarginal gyrus located in the left inferior parietal lobule. Thus, these regions are essential for any kind of vWM task (Paulesu et al., 1993; Smith and Jonides, 1998; Henson et al., 2000; Buchsbaum and D'Esposito, 2008). Moreover, parietal activation has been interpreted as a buffer for modality-specific information. Whereas, the relevance of prefrontal and parietal regions for vWM has long been recognized, the cerebellum came into focus only some years ago. Originally regarded mainly as a structure involved in motor control and coordination, its involvement in higher-order cognitive processes, such as vWM, is no longer called into question (Ravizza et al., 2006; Hayter et al., 2007; Cooper et al., 2012; Thürling et al., 2012; Tomlinson et al., 2014). More specifically, it has been suggested that the cerebellum plays a relevant role in subvocal rehearsal, but the specific contribution of the cerebellum to the various processes involved in vWM is still a matter of debate (Desmond et al., 2003; Pleger and Timmann, 2018). Like the cerebellum, the basal ganglia (BG) are critical structures for motor control by enhancing desired motor behaviors and suppressing undesired ones (Alexander et al., 1986; Mink, 1996). In addition, the BG are involved in various cognitive processes, such as language production and working memory (McNab et al., 2008). Again, for many years, fMRI studies on vWM tended to focus on cortical structures such as parietal and frontal regions, underestimating the relevance of BG structures such as caudate, putamen and globus pallidus. Finally, limbic areas, such as cingulate, are known to be involved in vWM, but its contribution has likewise long been underestimated (Moore et al., 2013).

\section{Influencing Factors in the Neural Correlates of vWM}

Activation in these brain regions can be influenced by several factors, such as age, gender, and type and difficulty (i.e., WM load) of the fMRI task. Moreover, the activation can be assumed to depend on individual performance (e.g., response velocity/speed as assessed by mean response times) and the statistical threshold which analyses are based on.

\section{Age}

Older adults compared to younger adults have been found to show a more bilateral pattern of prefrontal cortex activity under comparable task demands, a finding which constituted the basis of the Hemispheric Asymmetry Reduction in Older Adults (HAROLD) model (Cabeza, 2002; Cabeza et al., 2004). One hypothesis is that bilateral activity in older adults 
could reflect a functional compensatory mechanism, in which age-related asymmetry reductions compensate neurocognitive decline leading to a less lateralized brain activity. This is known as the compensation view. A second hypothesis is the so-called dedifferentiation view which assumes a less specific recruitment of neural networks due to gradual changes occurring with age. In a PET study, Reuter-Lorenz et al. (2000) showed that PFC activity in younger adults was left lateralized for verbal and right lateralized for spatial stimuli, whereas older adults presented a bilateral PFC activation for verbal and visual tasks. This model is not only supported by functional neuroimaging results but also by behavioral results from a letter matching task (Reuter-lorenz et al., 1999). Apart from these models, a number of other theories related to age differences in brain activation have been proposed, such as the Posterior-Anterior Shift in Aging (PASA). This theory assumes both frontal overactivation and posterior midline cortex under-activation in older adults compared to younger ones (Davis et al., 2009). The Compensation-Related Utilization of Neural Circuits Hypothesis (CRUNCH) proposes that people will activate more cortical regions if task difficulty increases (Reuter-Lorenz and Cappell, 2008). Finally, the Scaffolding Theory of Aging and Cognition (STAC and STAC-r) suggests that the increased frontal activation with age is a marker of the adaptive human brain indicating a compensation for the structural and functional decline going along with aging (Park and Reuter-Lorenz, 2009). This theory takes a holistic view by considering compensation a normal process involved in our daily lives in order to be able to achieve our goals.

\section{Gender}

The influence of gender in the context of WM and, more specifically, vWM, is still rather controversial, with some studies reporting no gender effects (Bell et al., 2006; Schmidt et al., 2009) and others reporting significant differences between male and female participants (Lejbak et al., 2011; Zilles et al., 2016). The controversial results might be due to the potential influence of sex hormones, which have been shown to influence several cognitive functions including vWM (Mordecai et al., 2008; Joseph et al., 2012). Sex hormones are known to fluctuate with, for instance, menstrual cycle or hormonal contraception. However, most studies did not provide any information on these aspects which may explain the result heterogeneity to some degree.

\section{Additional Factors (Tasks, Load, Mean, Reaction Time)}

A previous meta-analysis showed differences in brain activity due to WM task type (Rottschy et al., 2012). They found that n-back and Sternberg tasks, which are typical fMRI WM tasks, not only showed differences in mental processes but also in brain activation. Moreover, tasks can vary in their difficulty through modulating the WM load (i.e., the number of items that need to be remembered). Load effects reflect the neural activation related to the increasing memory demands of information (Cowan et al., 2012; Cowan, 2017). Rottschy et al. (2012) found that load effects were mainly associated with activation in the bilateral inferior frontal gyrus. Finally, Honey et al. (2000) demonstrated that prolonged mean reaction times (RT) in response to a vWM task could influence activation in WM related brain regions. Therefore, these findings suggest that all the previously mentioned potential factors should be taken into consideration.

\section{Aim of the Study}

Against this background, the first aim of the present study was to provide an updated and extended meta-analysis of the neural correlates of vWM in healthy humans using a coordinate-based meta-analysis. The second aim was to find out more about the role of the potential moderators (age, gender, type and difficulty of the fMRI task, mean RT, and statistical threshold). Although task performance which is related to the difficulty level could be another potential factor, it was not taken into consideration due to the heterogeneous assessment in the selected studies (i.e., absolute correct values, percentage of correct values, accuracy) as pointed also by Meule (2017). To our knowledge this is the first meta-analysis to study these factors in vWM. A better knowledge about their influence on the neural correlates of vWM will increase understanding of the general mechanisms of vWM as well as help to improve methods and analyses of future vWM studies.

\section{MATERIALS AND METHODS}

\section{Literature Search and Inclusion of Studies}

An exhaustive literature search was conducted on whole-brain fMRI studies on vWM from January 2000 to December 2017. We searched the databases PubMed, Scopus and Web of Science for English-language studies with the combination of the following key words: "n-back," "DMTS," "Sternberg," "delayed matched to sample," "delayed match to sample," plus "verbal working memory," "fMRI," "healthy." The Brainmap database was also searched with their respective search criteria (Subjects Size is more than 10, Experiments Paradigm Class is Delayed Match to Sample/n-back, Experiments Imaging Modality is fMRI, Conditions Stimulus is Visual Letters, and Subjects Handedness is Right). Further studies (11 publications) were identified through chasing citations from the selected studies (see Figure $\mathbf{1}$ for flowchart diagram). The "Meta-analysis of Observational Studies in Epidemiology" (MOOSE) guidelines Stroup et al. (2000) were used for the literature search and selection of studies. All articles were identified, selected and coded by a single investigator (M.E.). The same investigator double-checked the manually extracted peak coordinates and effect size values from the selected studies.

The criteria for inclusion were whole-brain analyses with reported results in a standard reference space (Talairach or MNI), inclusion of more than 10 healthy subjects and studies with clear boundaries between inclusion and exclusion criteria. Studies were excluded if they only included region-of-interests (ROI) analyses, did not report peak coordinates, investigated between- or within-group effects of pharmacological treatment, disease, living conditions, or used reward trials or emotional retrieval. We also excluded studies that re-analyzed previously reported data to avoid overestimating the effects. Positron emission tomography (PET) experiments were also not included in this meta-analysis due to methodological differences (e.g., 


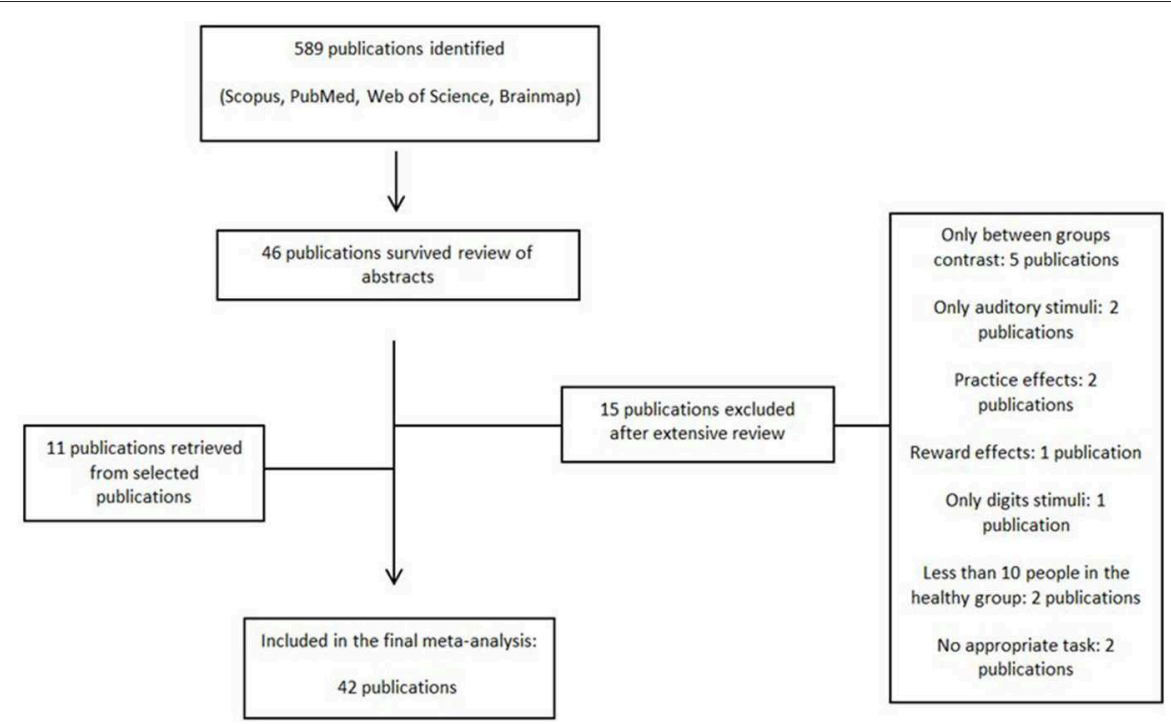

FIGURE 1 | Flowchart diagram of selected papers.

differences in temporal resolution between PET and MRI) and differences in the underlying physiology (i.e., BOLD contrast vs. glucose mechanism).

\section{Comprehensive Meta-Analysis}

We first conducted a meta-analysis of all the vWM studies. The demographic and study characteristics are shown in Table 1. The vast majority of the selected studies used the SPM software (https://www.fil.ion.ucl.ac.uk/spm/software/) to perform their fMRI analyses (83.3\% of studies) indicating a clear bias toward this software package. Coordinates and $t$-values included in the analysis are shown in Supplementary Table 1. When only $p$ or $z$-values were reported, they were transformed into $t$-values taking into account the sample size per study. The influence of gender (\% female), mean age, type of fMRI task (DMTS including the Sternberg task or n-back), mean RT, and the type of threshold used in the study (uncorrected vs. corrected) were studied with meta-regressions. The majority of studies corrected for multiple comparisons by controlling the false-discovery rate (FDR), except for one study that used the family-wise error rate (FWE) and another one that used Bonferroni correction. Those studies presenting results with peak coordinates at $p<0.005$ or $p<0.001$ uncorrected (33.3\%) controlled for the cluster-size with different thresholds $(5,8,10,17$, or 25 contiguous voxels). On a side note-although IQ and years of education have been shown to be associated with WM performance (Fukuda et al., 2010; Boller et al., 2017), we could not assess these factors here because the majority of studies did not provide any information on IQ or years of education.

There are several established fMRI vWM paradigms: nback, Sternberg, and delayed matching to sample (DMTS) tasks (Kirchner, 1958; Sternberg, 1966; Paule et al., 1998). N-back tasks include a sequential presentation of stimuli. Subjects have to decide whether the current stimulus is the same as the one $n$ positions before (e.g., the previous one in a 1-back condition or the one two positions back in a 2-back condition). In Sternberg tasks, a set of stimuli is presented simultaneously that need to be maintained over a certain period which is followed by a single probe stimulus for which participants need to decide whether it was part of the set or not. In DMTS tasks, a single stimulus is presented. After the maintenance period, a set of multiple probes is presented from which participants need to recognize the single stimulus they had to memorize. While n-back tasks are normally presented in the form of a block-design, DMTS and Sternberg tasks are presented in an event-related design.

\section{Load-Effect Meta-Analysis}

To assess the neural correlates of increasing vWM load (i.e., the difficulty of the fMRI task), we performed a load-effect metaanalysis. We only included studies in which there was a contrast between higher and lower vWM loads, such as 3-back vs. 1back or 3-back vs. 2-back. The selected studies are shown in Supplementary Table 2.

\section{Meta-Analytical Approach: ES-SDM}

We used the anisotropic effect-size version of seed-based d' mapping software (http://www.sdmproject.com) to conduct coordinate-based meta-analyses. The software uses a voxel-based meta-analytic approach. First, a strict selection of the reported peak coordinates of gray matter differences was applied by only including the studies containing whole-brain analyses. This is essential in order to avoid biased results from some neuroimaging studies, in which more liberal statistical thresholds were used for some ROIs relative to the rest of the brain. Peak coordinates in MNI or Talairach and effect size values were manually extracted from each contrast of interest in each study. All p- or z-values were transformed into $t$-values using SDM web utilities. Second, a map for the activation in gray matter was created for each 
TABLE 1 | Characteristics of the $42 \mathrm{fMRI}$ studies included in the meta-analysis.

\begin{tabular}{|c|c|c|c|c|c|c|c|c|c|}
\hline References & \multicolumn{3}{|c|}{ Demographic data } & $\%$ Fem & $\begin{array}{l}\text { fMRI task } \\
\text { Task }\end{array}$ & Contrast & Phases & $\begin{array}{l}\text { Mean RT } \\
\text { (ms) }\end{array}$ & $\begin{array}{l}\text { Mean accuracy } \\
(\%)\end{array}$ \\
\hline $\begin{array}{l}\text { Altamura et al., } \\
2007\end{array}$ & 18 & $27.4(\mathrm{NA})$ & NA & 38.9 & Sternberg & $\begin{array}{l}\text { Modulated by load and } \\
\text { delay }^{\dagger}, \text { load alone }{ }^{\ddagger}\end{array}$ & Block design & 999.2 & 87.83 \\
\hline $\begin{array}{l}\text { Bunge et al., } \\
2001\end{array}$ & 16 & $27.0(18-40)$ & NA & 18.8 & Sternberg & Load $6>\operatorname{load} 4$ & $\mathrm{E}, \mathrm{M}, \mathrm{R}$ & NA & 93 \\
\hline Cairo et al., 2004 & 18 & $27.5(\mathrm{NA})$ & NA & 55.6 & Sternberg & $\begin{array}{l}\text { Average across loads }{ }^{\dagger} \text {, } \\
\text { Linear regression with } \\
\text { load }^{\ddagger}\end{array}$ & $\mathrm{E}, \mathrm{M}, \mathrm{R}$ & NA & NA \\
\hline $\begin{array}{l}\text { Caseras et al., } \\
2006\end{array}$ & 12 & $33.5(24-45)$ & 7.1 & 66.7 & n-back & Modulated by load & & 635.8 & 89.83 \\
\hline $\begin{array}{l}\text { Deckersbach } \\
\text { et al., } 2008\end{array}$ & 17 & $25.6(\mathrm{NA})$ & 5.9 & 100 & n-back & $2>$ baseline & & 787.6 & 94.43 \\
\hline $\begin{array}{l}\text { Desmond et al., } \\
2003\end{array}$ & 13 & 55.6 (NA) & 11.3 & 0 & Sternberg & $\begin{array}{l}\text { High load }>\text { low load }(6 \\
\text { letters }>1 \text { letter) }\end{array}$ & $\mathrm{E}, \mathrm{M}, \mathrm{R}$ & NA & NA \\
\hline Dima et al., 2014 & 40 & 31.5 (NA) & 10.4 & 50 & n-back & $\begin{array}{l}1>\text { control, } 2>\text { control, } 3 \text { control }^{\dagger}\end{array}$ & & $\begin{array}{l}1: 596 \\
2: 659 \\
3: 748\end{array}$ & $\begin{array}{l}1: 100 \\
2: 91.2 \\
3: 72.8\end{array}$ \\
\hline $\begin{array}{l}\text { Garrett et al., } \\
2011\end{array}$ & 19 & $34.9(N A)$ & 12.5 & 31.6 & n-back & $1>$ control, $2>$ control $^{\dagger}$ & & 558.2 & 97.26 \\
\hline $\begin{array}{l}\text { Kirschen et al., } \\
2010\end{array}$ & 16 & $21.7(\mathrm{NA})$ & 6.0 & 31.3 & Sternberg & $\begin{array}{l}\text { High load }>\text { low load }(6 \\
\text { letters }>2 \text { letters) }\end{array}$ & $\mathrm{E}, \mathrm{M}, \mathrm{R}$ & NA & NA \\
\hline $\begin{array}{l}\text { Knops et al., } \\
2006\end{array}$ & 16 & $27.0(\mathrm{NA})$ & 7.7 & 0 & n-back & $2>1$ & & 983.5 & NA \\
\hline Lim et al., 2008 & 12 & $68.6(\mathrm{NA})$ & 6.2 & 58.3 & n-back & $1>$ baseline & & 650 & 96.9 \\
\hline $\begin{array}{l}\text { Lythe et al., } \\
2012\end{array}$ & 20 & 26.7 (NA) & 6.7 & 0 & n-back & $\begin{array}{l}\text { Activation with increasing } \\
\text { load }\end{array}$ & & 722 & 88.1 \\
\hline $\begin{array}{l}\text { Marquand et al., } \\
2008\end{array}$ & 20 & 43,7 (NA) & 8.3 & 65 & n-back & $2>$ control & & NA & NA \\
\hline $\begin{array}{l}\text { Marvel and } \\
\text { Desmond, } 2010\end{array}$ & 16 & $23.7(19-28)$ & NA & 62.5 & Sternberg & Task > baseline & $\mathrm{E}, \mathrm{M}, \mathrm{R}$ & NA & NA \\
\hline $\begin{array}{l}\text { McMillan et al., } \\
2007\end{array}$ & 14 & 25.6 (NA) & 3.6 & 64.3 & n-back & $\begin{array}{l}2>\text { control: identification, } \\
2>\text { control: color }{ }^{\dagger}\end{array}$ & & 1562.5 & 78 \\
\hline $\begin{array}{l}\text { McNab et al., } \\
2008\end{array}$ & 11 & $24(22-34)$ & 4.0 & 63.6 & Sternberg & Task > control & $\mathrm{E}, \mathrm{M}, \mathrm{R}$ & 1460 & 91.3 \\
\hline $\begin{array}{l}\text { Meisenzahl } \\
\text { et al., } 2006\end{array}$ & 12 & $33.6(22-48)$ & 9.27 & 8.3 & n-back & $2>$ control & & 752 & NA \\
\hline $\begin{array}{l}\text { Monks et al., } \\
2004\end{array}$ & 12 & 45.6 (NA) & 3.5 & 0 & Sternberg & All levels & $\mathrm{E}, \mathrm{M}, \mathrm{R}$ & 1080 & 90 \\
\hline
\end{tabular}


TABLE 1 | Continued

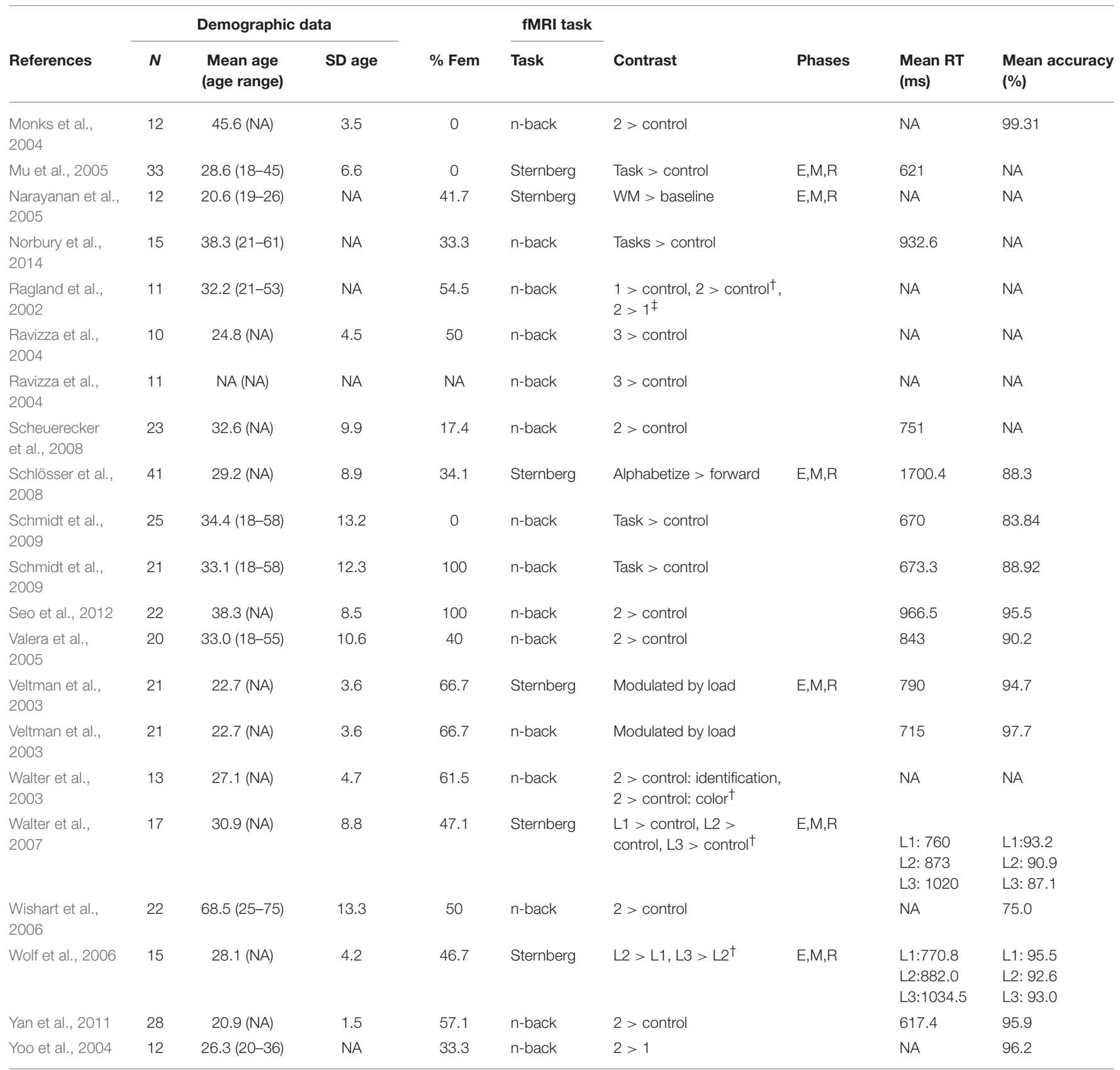

$n$, sample size; SD, standard deviation; NA, not announced; \% Fem, percentage of female participants; L, level; E, encoding; $M$, maintenance; R, recall; RT, reaction time.

${ }^{t}$ Combination of several contrasts into the final study contrast.

¥Contrast selected for the load-effect meta-analysis.

study using the Automated Anatomical Labeling (AAL) atlas partitioned into 116 brain regions (Tzourio-Mazoyer et al., 2002). If a study included more than one contrast of interest, we adjusted for multiple contrasts by combining the created images of each contrast into one image for the final analyses. The ES-SDM software re-creates the maps from the studies by converting the $t$-value of each peak to Hedge's g (Alegria et al., 2016). Third, an anisotropic non-normalized Gaussian kernel was applied by assigning different values to the different neighboring voxels based on the spatial correlation between them (Radua et al., 2014). At the end, we obtained a mean map by a voxelwise calculation of the mean of the study maps, weighted by the square root of the sample size, so that studies with larger sample sizes contributed more strongly (Radua and Mataix-Cols, 2009).

To assess the robustness of the main findings, we performed a whole-brain Jackknife analysis. Jackknife analysis consists of 


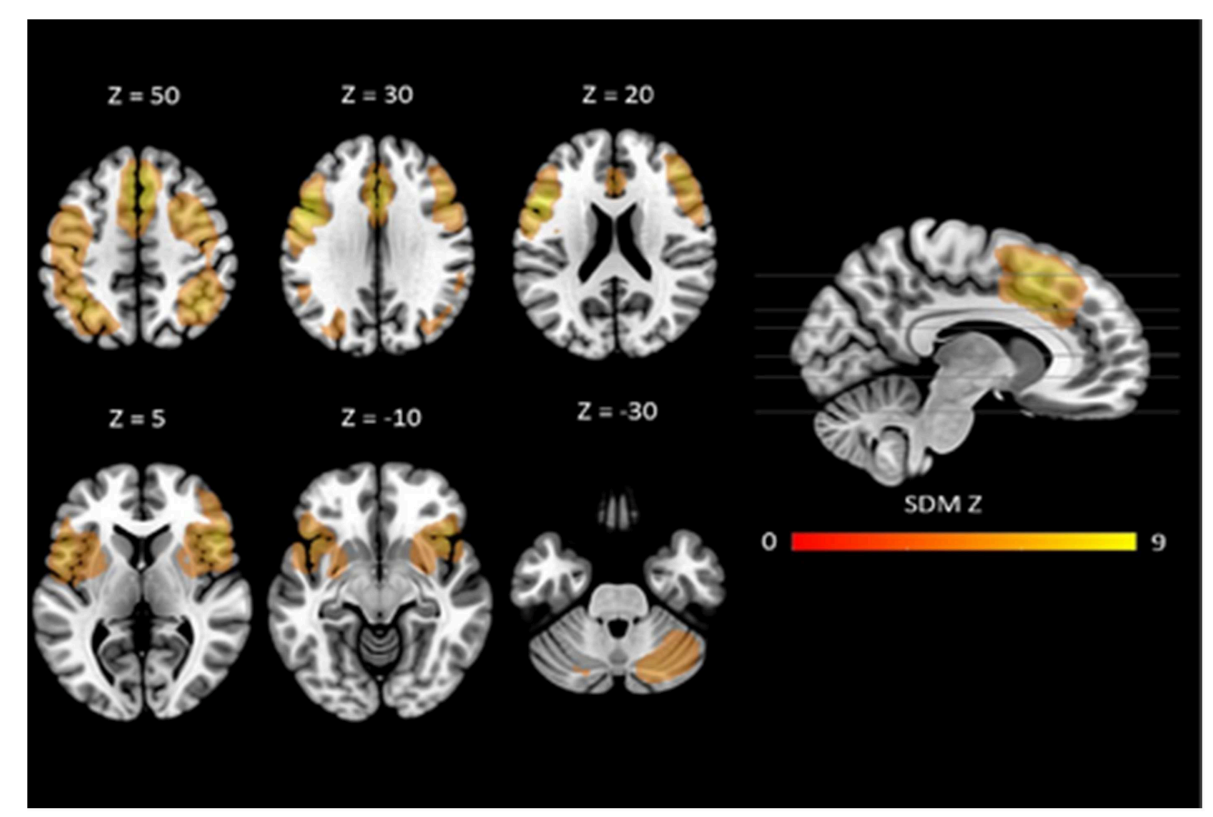

FIGURE 2 | Neural correlates of vWM estimated by meta-analysis. Results are displayed at $p<0.005$ (cluster size $\geq 10$ ) projected on the MNI 151 T1 template.

repeating the statistical analyses several times by discarding one study each time thus demonstrating the stability of the results (Müller et al., 2018). Heterogeneity of effect sizes and publication bias were assessed with the $I^{2}$ index and Egger's test (Egger et al., 1997; Müller et al., 2018). The $I^{2}$ index provides the proportion of variability across studies that is due to true heterogeneity relative to that from sampling error (Higgins and Thompson, 2002). Egger's tests were used to test for asymmetry of funnel plots, serving as an indicator of publication bias (see Supplementary Figure 1 for examples).

Statistical significance was determined with random-effects models. We used the default threshold for the calculated mean (voxel-level $p<0.005$ uncorrected, peak height threshold 1 , minimum cluster extent 10 contiguous voxels) (Radua and Mataix-Cols, 2009). To control for multiple testing in the several meta-regressions we used a more conservative threshold, Bonferroni-corrected threshold of $p<0.001$.

\section{RESULTS}

Comprehensive Meta-Analysis (42 Studies) The mean map of brain regions of the whole-brain metaanalysis for vWM is shown in Figure 2. The majority of studies reported only task-positive activation. We observed extended activation patterns in the frontal lobe including left superior frontal gyrus (SFG), medial frontal gyrus, right middle frontal gyrus (MFG), right inferior frontal gyrus (IFG), triangular, orbital and opercular part of the right IFG, orbital and opercular part of the left IFG, bilateral SMA, bilateral precentral gyrus, and left rolandic operculum. There was also activation in parietal areas including left post-central gyrus, right angular gyrus, and left inferior parietal gyri (IPG). Moreover, there was activation in the bilateral median cingulate, the left insula, the right lenticular nucleus (i.e., putamen and pallidum) and in bilateral cerebellum (crus I).

Robustness analyses showed that these results were preserved in all studies. Egger's tests indicated that there were some regions for which there was evidence of heterogeneity: left SFG, left SMA, left precentral gyrus, left post-central gyrus, right angular gyrus, left IPG, right median cingulate, left insula, and right cerebellum (crus I) (see Table 2).

Meta-regression analyses confirmed that mean age and mean RT moderated activation in some brain regions. Mean age was associated with decreased activation in the left rolandic operculum, left insula, left superior temporal gyrus (STG), left IFG (opercular part), left heschl gyrus, left post-central gyrus, left lenticular nucleus (putamen), and the right MFG. Mean RT was positively associated with activation in the left precentral gyrus and the left MFG (see Figure 3 and Table 3). None of the other meta-regression analyses yielded any significant results.

\section{Load-Effect Meta-Analysis (16 Studies)}

We found activation in several frontal areas: right SFG (dorsolateral and medial part), left SFG (medial part), right MFG, right IFG (triangular part), left IFG (triangular and opercular part), right SMA, bilateral precentral gyrus, bilateral rolandic operculum. Moreover, there was activation in several parietal areas (left post-central gyrus, left angular gyrus, left SPG, and bilateral IPG) as well as in the left anterior cingulate gyri, bilateral median cingulate gyri, left fusiform gyrus, and right cerebellum (crus I and hemispheric lobule VI) (see Table 4).

Jackknife analyses showed that the findings were preserved across studies, except for the right rolandic operculum and left fusiform gyrus, which were no longer detectable after discarding 
TABLE 2 | Comprehensive meta-analysis results.

\begin{tabular}{|c|c|c|c|c|c|c|c|}
\hline MNI coordinates & SDM-Z & $p$-value & Region & Voxels & $1^{2}$ & JK & Egger test ( $p$-value) \\
\hline$-50,12,28$ & 8.985 & $<0.00005$ & L. inferior frontal gyrus, opercular part & 758 & 51.98 & $45 / 45$ & 0.374 \\
\hline$-46,8,36$ & 8.831 & $<0.00005$ & L. precentral gyrus & 1807 & 58.65 & $45 / 45$ & 0.001 \\
\hline $4,18,44$ & 8.534 & $<0.00005$ & R. median cingulate / paracingulate gyri & 631 & 59.46 & $45 / 45$ & 0.015 \\
\hline $4,24,46$ & 8.483 & $<0.00005$ & R. supplementary motor area & 784 & 55.79 & $45 / 45$ & 0.051 \\
\hline $0,18,40$ & 8.359 & $<0.00005$ & L. superior frontal gyrus, medial & 772 & 55.76 & $45 / 45$ & 0.027 \\
\hline$-2,8,36$ & 8.322 & $<0.00005$ & L. median cingulate / paracingulate gyri & 510 & 4.06 & $45 / 45$ & 0.314 \\
\hline$-2,22,46$ & 8.214 & $<0.00005$ & L. supplementary motor area & 1166 & 62.59 & $45 / 45$ & 0.020 \\
\hline $50,26,2$ & 7.580 & $<0.00005$ & R. inferior frontal gyrus, triangular part & 1246 & 0.00 & $45 / 45$ & 0.732 \\
\hline $50,18,8$ & 7.397 & $<0.00005$ & R. inferior frontal gyrus, opercular part & 888 & 3.29 & $45 / 45$ & 0.168 \\
\hline $40,-58,44$ & 7.259 & $<0.00005$ & R. angular gyrus & 873 & 23.80 & $45 / 45$ & 0.001 \\
\hline $46,24,-6$ & 7.237 & $<0.00005$ & R. inferior frontal gyrus, orbital part & 401 & 2.47 & $45 / 45$ & 0.561 \\
\hline$-36,-54,48$ & 7.055 & $<0.00005$ & L. inferior parietal gyri & 1804 & 45.38 & $45 / 45$ & 0.000 \\
\hline $40,6,50$ & 6.917 & $<0.00005$ & R. precentral gyrus & 1297 & 0.00 & $45 / 45$ & 0.656 \\
\hline$-44,0,16$ & 6.293 & $<0.00005$ & L. rolandic operculum & 428 & 10.86 & $45 / 45$ & 0.386 \\
\hline $26,6,50$ & 5.911 & $<0.00005$ & R. middle frontal gyrus & 1604 & 0.00 & $45 / 45$ & 0.083 \\
\hline$-42,18,-6$ & 5.724 & $<0.00005$ & L. inferior frontal gyrus, orbital part & 446 & 54.76 & $45 / 45$ & 0.000 \\
\hline$-48,-22,46$ & 5.496 & $<0.00005$ & L. post-central gyrus & 1582 & 45.32 & $45 / 45$ & 0.002 \\
\hline$-36,8,0$ & 5.006 & $<0.00005$ & L. insula & 939 & 8.50 & $45 / 45$ & 0.019 \\
\hline $22,-76,-30$ & 4.683 & 0.000005 & R. cerebellum, crus I & 1186 & 45.55 & $45 / 45$ & 0.009 \\
\hline $32,0,-10$ & 4.249 & 0.000107 & R. lenticular nucleus, putamen & 577 & 0.77 & $45 / 45$ & 0.086 \\
\hline $24,0,-6$ & 4.167 & 0.000177 & R. lenticular nucleus, pallidum & 32 & & & \\
\hline$-20,-78,-30$ & 3.641 & 0.002827 & L. cerebellum, crus I & 36 & & & \\
\hline
\end{tabular}

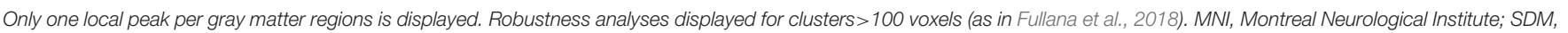
signed differential mapping; $I^{2}$, percentage of variance attributable to study heterogeneity; JK, jackknife sensitivity test; L., left; R., right.

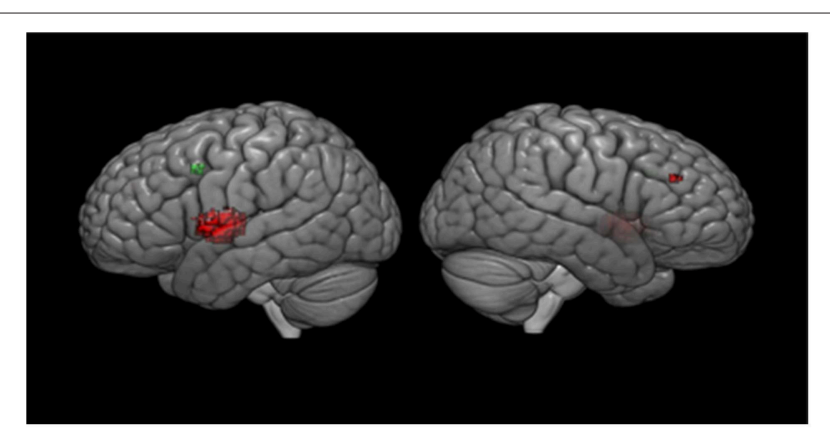

FIGURE 3 | Meta-regression results. Results are displayed at $p<0.001$ on MNI 152 2009. Red color, age regressor results; Green color, RT regressor results.

two papers. We only observed heterogeneity in the right inferior parietal gyrus (see Table 4).

\section{DISCUSSION}

The present comprehensive meta-analysis across 42 wholebrain vWM fMRI tasks showed vWM processing to be based on a fronto-parieto-cerebellar network and to involve also subcortical regions such as the cingulate, left insula and right lenticular nucleus. Thus, the present results corroborate previously discussed networks, but also provide evidence for the involvement of additional regions that have been neglected in the past in the discussion of vWM processing.

\section{Dual-Selection Model}

A tentative explanation of the results is provided by the dualselection model. Nee et al. (2013) proposed this model based on a meta-analysis of 36 event-related fMRI studies aimed at understanding the executive processes of WM. According to this model the caudal superior frontal sulcus (SFS) is associated with a spatial selection while the mid-lateral PFC is especially sensitive to non-spatial content, matching the "where" and "what" based selections, respectively. This proposal was further corroborated by a previous meta-analysis of 24 experiments based on an n-back task (Owen et al., 2005). The results of the present meta-analysis lend further support to the dual-selection model given that we also found activation of the mid-lateral PFC (bilateral IFG, right MFG, and medial part of the left SFG). The left SFG appeared to be a heterogeneous region. The fact that we found a noticeable bilateral prefrontal activation in this meta-analysis suggests that the assumption of a strongly left-lateralized verbal WM activation in PFC should be reconsidered. However, it needs to be clarified that we did not include studies systematically comparing spatial vs. non-spatial WM. Therefore, these conclusions need to be drawn with caution because the mere fact that 
TABLE 3 | Meta-regression analysis.

\begin{tabular}{|c|c|c|c|c|c|c|c|}
\hline \multirow[t]{2}{*}{ Mean_age } & \multirow{2}{*}{$\begin{array}{l}\text { Clusters showing a negative } \\
\text { correlation with age }\end{array}$} & \multicolumn{4}{|c|}{ Maximum } & \multicolumn{2}{|l|}{ Cluster } \\
\hline & & SDM value & $p$-value & Voxels & Description & Breakdown & Voxels \\
\hline & $-48,-4,8$ & -2.387 & $<0.00005$ & 1204 & L. rolandic operculum & L. rolandic operculum & 415 \\
\hline & & & & & & L. insula & 332 \\
\hline & & & & & & $\begin{array}{l}\text { L. superior temporal } \\
\text { gyrus }\end{array}$ & 175 \\
\hline & & & & & & $\begin{array}{l}\text { L. inferior frontal gyrus, } \\
\text { opercular part }\end{array}$ & 115 \\
\hline & & & & & & L. heschl gyrus & 47 \\
\hline & & & & & & L. post-central gyrus & 34 \\
\hline & & & & & & $\begin{array}{l}\text { L. temporal pole, } \\
\text { superior temporal gyrus }\end{array}$ & 16 \\
\hline & & & & & & $\begin{array}{l}\text { L. lenticular nucleus, } \\
\text { putamen }\end{array}$ & 13 \\
\hline & & & & & & L. precentral gyrus & 1 \\
\hline & & & & & & (undefined) & 56 \\
\hline & $48,38,24$ & -1.736 & 0.00028 & 16 & $\begin{array}{l}\text { R. inferior frontal gyrus, } \\
\text { triangular part }\end{array}$ & R. middle frontal gyrus & 10 \\
\hline & & & & & & $\begin{array}{l}\text { R. inferior frontal gyrus, } \\
\text { triangular part }\end{array}$ & 6 \\
\hline \multirow[t]{4}{*}{ Mean_RT } & $\begin{array}{l}\text { Clusters showing a positive } \\
\text { correlation with RT }\end{array}$ & Maximum & & & & Cluster & \\
\hline & MNI Coordinates & SDM value & $p$ value & Voxels & Description & Breakdown & Voxels \\
\hline & $-46,10,42$ & 3.949 & 0.00028 & 29 & L. precentral gyrus & L. middle frontal gyrus & 17 \\
\hline & & & & & & L. precentral gyrus & 12 \\
\hline
\end{tabular}

MNI, Montreal Neurological Institute; SDM, signed differential mapping, R., right; L., left.

we found the same activation does not fully support the dual-selection model.

\section{Phonological Loop}

We did not find activation of the left supramarginal gyrus, which is known to be important for the phonological store, but instead in the region where the supramarginal gyrus is located, the left inferior parietal cortex. The activation of this region was found to be heterogeneous, which tends to be in line with the hypothesis of Buchsbaum and D'Esposito (2008). They argue that the phonological store does not precisely correspond to a single specific functional brain region, but rather is associated with several brain regions that underlie neural processes from perception and production of speech. Surprisingly, the present meta-analysis did not reveal any activation in the Wernicke area although this is an essential area for the comprehension and/or production of verbal material (Binder, 2015). This area is assumed to comprise mainly the posterior part of the superior temporal gyrus as well as the occipito-parieto-temporal junction including the angular gyrus. However, the exact location of the Wernicke area is still a matter of debate also due to its comprehensive and partly heterogeneous functionality in the context of verbal processing. Moreover, the fact that we used the AAL atlas, which comprises relatively large brain regions, might also explain why we were not able to isolate activation of this specific and somewhat ill-defined region. The fact that we did not find any activation in the right parietal cortex was also expected, since this region is assumed to serve spatial rehearsal. Hence, as opposed to the bilateral activation in the prefrontal cortex, activation in the parietal cortex turned out to be strongly left-lateralized, presumably due to modality. As predicted, we also found activation in the left IFG containing the Broca's area, as well as in the left SMA, which are components central to the articulatory loop. The fact that we did not find any activation in the premotor cortex could also be due to the atlas used. The AAL atlas does not contain this region because the labeled SMA embeds both the premotor cortex and the pre-SMA. Therefore, we cannot exclude that there was specific activation of the premotor cortex. In addition, there was activation in the left rolandic operculum, which is caudally adjacent to Broca's area. It has been demonstrated that this brain area is involved in speech production (Koelsch et al., 2009) and speech prosody processing (Wu et al., 2017). To the best of our knowledge, this is the first WM metaanalysis isolating activation specifically in this area. This finding may indicate that the majority of the participants used an overt rehearsal strategy during the vWM tasks. Further studies testing the impact of the opportunity for rehearsal during vWM tasks on brain activation are however needed to confirm this hypothesis. 
TABLE 4 | Load-effect meta-analysis results.

\begin{tabular}{|c|c|c|c|c|c|c|c|}
\hline MNI coordinates & SDM-Z & $p$-value & Region & Voxels & $1^{2}$ & JK & Egger test ( $p$-value) \\
\hline$-46,8,38$ & 5.617 & $<0.0000001$ & L. precentral gyrus & 1315 & 52.15 & $15 / 15$ & 0.152 \\
\hline $46,34,18$ & 5.502 & $<0.0000001$ & R. middle frontal gyrus & 1378 & 31.70 & $15 / 15$ & 0.339 \\
\hline $8,32,48$ & 5.442 & $<0.0000001$ & R. superior frontal gyrus, medial & 387 & 5.78 & $15 / 15$ & 0.828 \\
\hline$-48,14,26$ & 5.395 & $<0.0000001$ & L. inferior frontal gyrus, triangular part & 1211 & 55.42 & $15 / 15$ & 0.107 \\
\hline$-50,16,22$ & 5.356 & $<0.0000001$ & L. inferior frontal gyrus, opercular part & 757 & 47.91 & $15 / 15$ & 0.170 \\
\hline $8,24,48$ & 5.231 & $<0.0000001$ & R. supplementary motor area & 452 & 29.95 & $15 / 15$ & 0.741 \\
\hline $0,28,50$ & 5.201 & $<0.0000001$ & L. superior frontal gyrus, medial & 892 & 38.10 & $15 / 15$ & 0.864 \\
\hline$-2,6,36$ & 4.902 & $<0.0000001$ & L. median cingulate / paracingulate gyri & 429 & 7.39 & $15 / 15$ & 0.746 \\
\hline $4,6,38$ & 4.879 & $<0.0000001$ & R. median cingulate / paracingulate gyri & 587 & 0.00 & $15 / 15$ & 0.831 \\
\hline$-40,-58,46$ & 4.793 & $<0.0000001$ & L. angular gyrus & 120 & 0.00 & $15 / 15$ & 0.415 \\
\hline$-2,8,30$ & 4.686 & $<0.0000001$ & L. anterior cingulate / paracingulate gyri & 633 & 4.28 & $15 / 15$ & 0.687 \\
\hline $50,30,4$ & 4.646 & $<0.0000001$ & R. inferior frontal gyrus, triangular part & 1228 & 0.59 & $15 / 15$ & 0.534 \\
\hline$-38,-48,44$ & 4.563 & 0.00000001 & L. inferior parietal gyri & 961 & 39.39 & $15 / 15$ & 0.487 \\
\hline $22,-80,-30$ & 4.248 & 0.00000101 & R. cerebellum, crus I & 1443 & 8.61 & $15 / 15$ & 0.883 \\
\hline $40,-46,48$ & 4.143 & 0.00000179 & R. inferior parietal gyri & 682 & 62.41 & $15 / 15$ & 0.001 \\
\hline $22,14,56$ & 3.676 & 0.00002283 & R. superior frontal gyrus, dorsolateral & 94 & & & \\
\hline$-50,-16,42$ & 3.640 & 0.00002819 & L. post-central gyrus & 688 & 1.97 & $15 / 15$ & 0.790 \\
\hline $52,10,-2$ & 3.434 & 0.00009483 & R. rolandic operculum & 144 & 11.73 & $14 / 15$ & 0.700 \\
\hline $30,-50,-34$ & 3.401 & 0.00011838 & R. cerebellum, hemispheric lobule VI & 704 & 7.75 & $15 / 15$ & 0.974 \\
\hline$-50,-6,14$ & 3.354 & 0.00015760 & L. rolandic operculum & 347 & 0.20 & $15 / 15$ & 0.827 \\
\hline$-26,-60,54$ & 3.242 & 0.00029481 & L. superior parietal gyrus & 255 & 0.00 & $15 / 15$ & 0.343 \\
\hline $46,-10,46$ & 3.160 & 0.00046253 & R. precentral gyrus & 348 & 0.00 & $15 / 15$ & 0.624 \\
\hline$-32,-76,-16$ & 2.933 & 0.00154328 & L. fusiform gyrus & 320 & 0.55 & $14 / 15$ & 0.725 \\
\hline
\end{tabular}

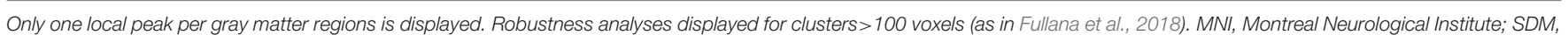
signed differential mapping; ${ }^{2}$, percentage of variance attributable to study heterogeneity; JK, jackknife sensitivity test; R., right; L., left.

\section{Attentional Control}

Many conceptualizations of WM include an attentional control mechanism that supports dealing with interferences such as from other items in memory. The right IFG has been proposed to be an important region for attentional control (Aron et al., 2003; Forstmann et al., 2008). Specifically, Aron et al. 's (2003) data strongly suggest that response inhibition is uniquely located in the right IFG, in particular in its triangular part. The data were acquired by studying patients with lesions of the right frontal lobe during a go/no-go task. Forstmann et al. (2008) found a direct linkage between structural and functional properties of the right IFG, and its role in response inhibition. Another fMRI study (Aron and Poldrack, 2006) found that the IFG targets the subthalamic nucleus (STN) and regions in its vicinity. The STN sends excitatory projections to the globus pallidus externus, which, in turn, suppresses the thalamo-cortical output; this is assumed to lead to an inhibition of the initiated response. Finally, a strongly right-lateralized network comprising the right IFG, the STN, and also the pre-SMA, is recruited during response suppression (Aron, 2007). It remains unclear, however, whether the right IFG triggers the STN directly or via the preSMA (Aron et al., 2014). We found a strong activation of the right IFG, especially in the triangular part, the right SMAwhich also includes the pre-SMA in this atlas -, and the right pallidum, giving support to the idea that these areas constitute a network subserving response inhibition in the context of vWM processing. Indeed, a substantial body of behavioral research has found that attentional control as employed in response inhibition tasks is related to WM capacity (Kane and Engle, 2003; Unsworth and Engle, 2007, but see Rey-Mermet et al., 2019). Notably, heterogeneity analyses confirmed the stability of these networks indicating that activation in these regions is not likely due to a possible publication bias. However, it is important to mention that the selected studies did not manipulate attention. The fact that we found activation in the same areas that mediate response inhibition in other experimental contexts does not completely mean that they do so in the context of vWM.

In addition to the IFG, the angular gyrus has been found to be activated in the context of response inhibition (Wager et al., 2005). The angular gyrus is located in the posterior part of the inferior parietal lobule and has been found to be activated in a variety of tasks (Seghier, 2013). Some anatomical studies (Makris et al., 2005, 2009; Uddin et al., 2010) define the angular gyrus as an important seed point, given its strong interaction with temporo-frontal subsystems as well as regions such as hippocampus, caudate, and precuneus. It is a key component of the default-mode network and shows activation in most tasks demanding information retrieval (Spaniol et al., 2009; Kim, 2010). The role of this region in memory retrieval is plausible given its strong connectivity with the hippocampus. To the best of our knowledge, this is the first time that the right angular gyrus appears in a vWM meta-analysis. Considering that this region 
has been reported to be important for inhibition and retrieval we conclude that the activation of the angular gyrus in the present meta-analysis may predominantly reflect the employment of attentional control during information retrieval. Although we cannot exclude the possibility of this region's activity being found due to presence of publication bias in the selected literature. Further studies allowing for a separate analysis of the retrieval process are however needed to further explore this assumption.

\section{Cerebellar and Subcortical Activations}

It has been shown that cerebellum is connected not only to motor areas, but also to prefrontal cortical areas (Schmahmann, 1996); this suggests an involvement of the cerebellum in higher-order cognitive processes. A distinct cross-cerebro-cerebellar circuitry for vWM has been proposed with predominant involvement of right cerebellum, especially the lobule VI ( $\mathrm{Ng}$ et al., 2016). In accordance with this proposal, earlier studies already pointed at the relevance of the right cerebellum in the context of vWM. Using inhibitory continuous theta burst stimulation (cTBS) Tomlinson et al. (2014) found that participants were less accurate during a verbal version of the Sternberg task if a trial was preceded by a stimulation of the right cerebellar hemisphere. Moreover, patients with right-sided cerebellar lesions have been found to be impaired in verbal memory, whereas patients with left cerebellar lesions turned out to be slower in a visuospatial task (Hokkanen et al., 2006). All these findings suggest a lateralized function of the cerebellum with its right hemisphere contributing mainly to verbal and its left hemisphere to visuospatial processing. Moreover, a meta-analysis (Stoodley and Schmahmann, 2009) analyzing cerebellum neuroimaging studies found that regions involved in vWM studies overlap with those involved in language tasks which is in agreement with domain-specific storage modules as in Baddeley's model. It corroborates the idea that $\mathrm{vWM}$ is more right-lateralized with a strong activation occurring mostly at the junction lobule VI/Crus I. Our results showing a significantly stronger activation in the right cerebellum (crus I) support this hypothesis. A case study of a right cerebellar hemispherectomy in an 18-years-old patient reported that the patient suffered from a disproportionate impairment of the rehearsal system, while the phonological store was preserved (Silveri et al., 1998). This could be due to anatomical connections between Broca's area, left SMA, right lobule VI and crus I of the cerebellum (Schmahmann, 1991). However, in the present meta-analysis, we did not differentiate between those processes and, thus, we cannot further investigate whether the right cerebellum is mainly involved in rehearsal. Still, our analysis provides clear evidence for the relevance of the right cerebellum, especially crus I, in the context of vWM processing. Further studies disentangling the different vWM processes are warranted to elucidate the specific function of the right cerebellum in vWM.

Apart from the cerebellum, a number of additional subcortical areas are assumed to be relevant for vWM. Thus, basal ganglia regions, especially the caudate and the lenticular nucleus, have been found to be activated during encoding and maintenance phases during vWM tasks (Lewis et al., 2004; Chang et al., 2007; Moore et al., 2013). Although in the present meta-analysis basal ganglia activation was restricted to the right lenticular nucleus, it remains unclear whether the activation is ascribable to these processes. Again, we were unable to distinguish between the different vWM processes given the available data. Caudate, putamen and capsular regions are known to receive afferents from the left pre-SMA region, which is involved in vWM (Inase et al., 1999). Crosson et al. (2003) found that basal ganglia activity was accompanied by activation of the left pre-SMA during a word production task. They hypothesized that the increase of right basal ganglia activation serves to suppress the non-dominant right frontal cortex, whereas the increase of the left basal ganglia activation serves to enhance the language processing of the left dominant hemisphere. Against the background of these findings, the basal ganglia can be assumed to interact closely with the frontal cortex and to serve as a selective gating mechanism for the prefrontal cortex (Frank et al., 2001). From this perspective, the findings showing the basal ganglia to be active only during encoding and maintenance phases seem plausible, because selective gating plays a major role for these processes. However, as we did not study the phases separately, we cannot rule out that these activations also reflect attentional processes in addition to pure vWM processes. Moreover, six publications compared activation conditions with a simple baseline (e.g., fixation of a cross hair). Hence, we cannot rule out that some parts of the subcortical activation were due to motor activity (i.e., button press in the activation conditions vs. no button press in the baseline condition). In the present meta-analysis we also found left pre-SMA activation, but a conclusion about their influence on basal ganglia is unwarranted without any connectivity data. In addition to the pre-SMA activation, our meta-analysis demonstrated significant activation in the anterior cingulate which has been found to be activated during vWM tasks before (Bedwell et al., 2005; Narayanan et al., 2005). It should be noted that in the AAL atlas the significant cluster was labeled as median cingulate, which is part of the anterior cingulate. It is striking, however, that a majority of vWM did not find an involvement of the anterior cingulate. Hence, future studies should make an attempt to clarify the specific contribution of the different parts of the cingulate to vWM.

\section{Age, Load, and Mean RT as Influencing Factors}

Age-related changes in vWM are not fully understood because of a lack of longitudinal data. A recent longitudinal study found the activation of left prefrontal cortex (i.e., MFG and parts of the IFG) to be reduced during a vWM manipulation task in older people (Rieckmann et al., 2017). Somewhat in accordance with this finding, the present meta-analysis demonstrated a negative association between activation in the left and right IFG-including Broca's area-and age. In addition, we found a negative association between activation in the right MFG and age. It is known that cortical thickness, surface area, and volume of this region decrease with age (Lemaitre et al., 2012) which may, to some degree, explain this finding. Moreover, the right MFG plays a central role in reorienting attention from exogenous to endogenous attentional control (Japee et al., 2015). 
Our results are in agreement with the ontogenetic model of brain development according to which those brain regions that are the last to mature are the first to be affected by aging (Raz et al., 2005). Of note, all the other regions exhibiting a negative association between activation and age were localized in the left hemisphere. We found this negative association in the left insula, which-as stated above-plays a relevant role in the context of rehearsal, in the left putamen, which is involved in the active filtering of irrelevant material allowing us to focus on relevant material (Moore et al., 2013) in the left rolandic operculum, important for overt rehearsal, and in the left superior temporal gyrus, parts of which are critically involved in phonological storage. The fact that these regions important for different vWM processes showed a negative association with age might explain why older people tend to exhibit worse vWM performance, although it should be kept in mind that we did not take into account any longitudinal data or individual subject performance. Since these associations were detectable mainly in the left hemisphere and age-related changes were not restricted to the right PFC, our results seem to speak against the HAROLD model (Cabeza et al., 2002). Overall, the present results do not provide any evidence for a decrease of this lateralization with age, as claimed in other studies (Reuter-Lorenz et al., 2000; Cabeza et al., 2002, 2004). The fact that we found a decreased frontal activation with increasing age could either mean that the brain is not as adaptive as proposed by the earlier discussed STAC model or indicate that task demands were too high for elderly people leading to a "breakdown" of frontal activation instead of a compensatory increase. In order to draw any further conclusion it would be helpful to study the activation of these regions in elderly people taking also into account their individual task performance (e.g., accuracy). However, only three studies selected for the metaanalysis specifically included older populations; thus, the agerange was clearly undersampled in the current meta-analysis and the power to reliably assess the influence of age was too low. This might also explain why our results do not seem to be in line with the HAROLD model. Therefore, more empirical data comparing older and younger populations are necessary in order to find out more about specific age differences in activation during vWM. A better understanding of these age-related differences would pave the way for creating more sophisticated methods to preserve or enhance cognitive function in elderly populations.

Höller-Wallscheid et al. (2017) hypothesized the decreased lateralization across the PFC to be independent of age, but to depend on the subjective difficulty of WM tasks. In line with this hypothesis we found a bilateral activation across the PFC in the load effect meta-analysis. Our results are also in accordance with the load effect meta-analysis performed by Rottschy et al. (2012). The CRUNCH model states that the extent of cortical activation depends on the task load. Our results support this model, since we found a positive correlation between activation in several cortical regions (e.g., frontal areas) and task load. Apart from PFC areas we also found activation in the parietal cortex (IPG and left SPG) to be influenced by load, as reported in a previous study by Braver et al. (1997). Likewise, activation of the right lobule VI and crus I of the cerebellum turned out to depend on the difficulty of the vWM tasks. This is in accordance with a previous study which showed these parts of the cerebellum to respond to changes in vWM load (Kirschen et al., 2005). As stated before, there are anatomical connections between these parts of the cerebellum and frontal areas. Hence, the increased input from frontal regions involved in the articulatory system during a load manipulation could also reflect the increased activation of the right cerebellum. In addition, we found an association between load and activation in the fusiform gyrus. Tsapkini and Rapp (2010) pointed out that lesions of the left fusiform gyrus were significantly associated with reading and spelling deficits. In light of this finding, the positive correlation between load and activation in the left fusiform gyrus in the present study might indicate that a majority of people may have used overt rehearsal as a strategy to cope with increasing task difficulty.

It has long been recognized that RT is sensitive to manipulations of any kind of WM load (Just and Carpenter, 1992). Therefore, RT can also be viewed as a measure of load. A previous study found a significant positive correlation between RT and fMRI signal in nine subjects in the MFG and the left IFG (Braver et al., 1997). The present meta-analysis partially corroborates these findings showing both the left MFG and the left precentral gyrus to be positively associated with RT. However, our results seem to contradict a study by Honey et al. (2000), which reported that posterior parietal cortical activation was predicted by a prolonged RT in a vWM task. Importantly though, as states earlier, activation in the parietal cortex is influenced by load, which-in turn is related to RT. Moreover, the present meta-analysis revealed a positive association between left precentral gyrus activation and RT. This finding is plausible considering that the left precentral gyrus constitutes a major part of the primary motor area and its activation is contralateral to the side of the hand movement. Hence, increased activation in the primary motor cortex might facilitate faster responding. It should be emphasized that RT information was not available for all studies. Moreover, RT depends on many other factors such as number of responses alternatives, type of discrimination or delay time. Therefore, results of this factor should be treated with caution.

Although we performed a meta-analysis in which only fMRI studies were included, there has been a previous meta-analysis in which they selected both FMRI and PET studies to isolate the neural correlates of human working memory (Wager and Smith, 2003). Although they found some support for left frontal cortex dominance in vWM tasks, this was only for tasks with low executive demand. These results support our finding regarding the lateralization, i.e., the higher the difficulty on the task, the less lateralization of PFC activation is to be expected.

Finally, we expected the type of fMRI paradigm to be a significant moderator as demonstrated in a previous metaanalysis (Rottschy et al., 2012). This expectation was not met by the data. This could be due to a strong overlap in task activation, with potentially existing subtle quantity differences being too weak to be significant. We also found that gender did not affect activation associated with vWM tasks. However, we cannot exclude that gender differences would emerge when controlling for effects of sex hormones. Hence, future studies are required that carefully consider these potentially confounding factors. 


\section{Limitations}

In the present meta-analysis we discussed a number of relevant networks based on fMRI activity, such as the attentional system, but we did not take into account brain connectivity. However, the localization of brain areas is just the first step toward a more comprehensive understanding of the neural correlates of vWM. Analyses based on temporal dynamics, such as EEG or single-unit recordings, are essential to build a more integrative view. Another limitation regards our cerebellum findings. There is strong reason to assume that we did not find any inferior cerebellum activation because some of the scans included in the present meta-analysis did not cover the whole cerebellum due to methodological limitations (e.g., trade-off between brain coverage and repetition time).

\section{CONCLUSIONS}

We used a coordinate-based meta-analysis to integrate the current literature on $\mathrm{vWM}$ in healthy humans. We found activation of the established fronto-parietal network and the right cerebellum, especially crus I, and lobule VI. Our results support the dual-selection model, according to which a midlateral PFC activation occurs due to verbal input. Moreover, our results illustrate that we should not underestimate the activation of subcortical regions that play an important role for response inhibition. Age, mean RT, and load moderate vWM task activation and, thus, should be taken into consideration in future research. Especially the influencing factor of age should be further analyzed since the sample included in the present meta-analysis consists of primarily young people. Mean reaction time, moreover, could be influenced by many other

\section{REFERENCES}

Alegria, A. A., Radua, J., and Rubia, K. (2016). Meta-analysis of fmri studies of disruptive behavior disorders. Am. J. Psychiatry 173, 1119-1130. doi: 10.1176/appi.ajp.2016.15081089

Alexander, G. E., DeLong, M. R., and Strick, P. L. (1986). Parallel organization of functionally segregated circuits linking basal ganglia and cortex. Annu. Rev. Neurosci. 9, 357-381. doi: 10.1146/annurev.ne.09.030186.002041

Altamura, M., Elvevåg, B., Blasi, G., Bertolino, A., Callicott, J. H., Weinberger, D. R., et al. (2007). Dissociating the effects of Sternberg working memory demands in prefrontal cortex. Psychiatry Res. Neuroimaging 154, 103-114. doi: 10.1016/j.pscychresns.2006.08.002

Aron, A. R. (2007). The neural basis of inhibition in cognitive control. Neuroscientist 13, 214-228. doi: 10.1177/1073858407299288

Aron, A. R., Fletcher, P. C., Bullmore, E. T., Sahakian, B. J., and Robbins, T. W. (2003). Stop-signal inhibition disrupted by damage to right inferior frontal gyrus in humans. Nat. Neurosci. 6, 115-116. doi: 10.1038/nn1003

Aron, A. R., and Poldrack, R. A. (2006). Cortical and subcortical contributions to stop signal response inhibition: role of the subthalamic nucleus. J. Neurosci. 26, 2424-2433. doi: 10.1523/JNEUROSCI.4682-05.2006

Aron, A. R., Robbins, T. W., and Poldrack, R. A. (2014). Inhibition and the right inferior frontal cortex: one decade on. Trends Cogn. Sci. 18, 177-185. doi: 10.1016/j.tics.2013.12.003

Baddeley, A. (2000). The episodic buffer : a new component of working memory? Trends Cogn. Sci. 4, 417-423. doi: 10.1016/S1364-6613(00)01538-2

Baddeley, A. (2010). Working memory. Curr. Biol. . 20, 136-140. doi: 10.1016/j.cub.2009.12.014 factors. Further, more fine-grained studies are needed to gain a better understanding of the neural correlates underlying processes involved in vWM including encoding, maintenance, and retrieval.

\section{AUTHOR CONTRIBUTIONS}

$\mathrm{ME}, \mathrm{CB}$, and $\mathrm{KK}$ contributed to the conception and design of the study. ME selected the analyzed studies, performed the statistical analysis, and wrote the first draft of the manuscript. $\mathrm{CB}$ and $\mathrm{KK}$ wrote sections of the manuscript. All authors contributed to manuscript revision, read and approved the submitted version.

\section{FUNDING}

This work was supported by a Deutsche Forschungsgemeinschaft (DFG) grant to KK [grant number KO 3744/8-1].

\section{ACKNOWLEDGMENTS}

The authors wish to thank the users and creators of SDM software. Parts of this work have been presented at the 11th FENS Forum 2018 in Berlin, Germany and at the 8th IMPRS NeuroCom Summer School in Leipzig, Germany.

\section{SUPPLEMENTARY MATERIAL}

The Supplementary Material for this article can be found online at: https://www.frontiersin.org/articles/10.3389/fnhum. 2019.00180/full\#supplementary-material
Baddeley, A. D., and Hitch, G. (1974). "Working memory," in Psychology of Learning and Motivation Bower, Vol. 8, ed H. B. T Gordon (New York, NY: Academic Press), 47-89. doi: 10.1016/S0079-7421(08)60452-1

Bedwell, J. S., Horner, M. D., Yamanaka, K., Li, X., Myrick, H., Nahas, Z., and George, M. S. (2005). Functional neuroanatomy of subcomponent cognitive processes involved in verbal working memory. Int. J. Neurosci. 115, 1017-1032. doi: 10.1080/00207450590901530

Bell, E. C., Willson, M. C., Wilman, A. H., Dave, S., and Silverstone, P. H. (2006). Males and females differ in brain activation during cognitive tasks. Neuroimage 30, 529-538. doi: 10.1016/j.neuroimage.2005.09.049

Binder, J. R. (2015). The Wernicke area Modern evidence and a reinterpretation. Neurology 85, 2170-2175. doi: 10.1212/WNL.00000000000 02219

Boller, B., Mellah, S., Ducharme-Lalibert,é, G., and Belleville, S. (2017). Relationships between years of education, regional grey matter volumes, and working memory-related brain activity in healthy older adults. Brain Imaging Behav. 11, 304-317. doi: 10.1007/s11682-016-9621-7

Braver, T. S., Cohen, J. D., Nystrom, L. E., Jonides, J., Smith, E. E., and Noll, D. C. (1997). A parametric study of prefrontal cortex involvement in human working memory. Neuroimage 5, 49-62. doi: 10.1006/nimg.1996.0247

Buchsbaum, B. R., and D'Esposito, M. (2008). The search for the phonological store: from loop to convolution. J. Cogn. Neurosci. 20, 762-778. doi: 10.1162/jocn.2008.20501

Buchsbaum, B. R., Padmanabhan, A., and Berman, K. F. (2011). The neural substrates of recognition memory for verbal information: spanning the divide between short- and long-term memory. J. Cogn. Neurosci. 23, 978-991. doi: 10.1016/j.asieco.2008.09.006.EAST 
Bunge, S. A., Ochsner, K. N., Desmond, J. E., Glover, G. H., and Gabrieli, J. D. E. (2001). Prefrontal regions involved in keeping information in and out of mind. Brain 124, 2074-2086. doi: 10.1093/brain/124.10.2074

Cabeza, R. (2002). Prefrontal and medial temporal lobe contributions to relational memory in young and older adults. Psychol. Aging 17, 85-100. doi: 10.1037//0882-7974.17.1.85

Cabeza, R., Daselaar, S. M., Dolcos, F., Prince, S. E., Budde, M., and Nyberg, L. (2004). Task-independent and task-specific age effects on brain activity during working memory, visual attention and episodic retrieval. Cereb. Cortex 14, 364-375. doi: 10.1093/cercor/bhg133

Cabeza, R., Dolcos, F., Graham, R., and Nyberg, L. (2002). Similarities and differences in the neural correlates of episodic memory retrieval and working memory. Neuroimage 16, 317-330. doi: 10.1006/nimg.2002.1063

Cairo, T. A., Liddle, P. F., Woodward, T. S., and Ngan, E. T. C. (2004). The influence of working memory load on phase specific patterns of cortical activity. Cogn. Brain Res. 21, 377-387. doi: 10.1016/j.cogbrainres.2004.06.014

Caseras, X., Mataix-Cols, D., Giampietro, V., Rimes, K. A., Brammer, M., Zelaya, F., et al. (2006). Probing the working memory system in chronic fatigue syndrome: a functional magnetic resonance imaging study using the n-back task. Psychosom. Med. 68, 947-955. doi: 10.1097/01.psy.0000242770.50979.5f

Chai, W. J., Abd Hamid, A. I., and Abdullah, J. M. (2018). Working memory from the psychological and neurosciences perspectives: a review. Front. Psychol. 9:401. doi: 10.3389/fpsyg.2018.00401

Chang, C., Crottaz-Herbette, S., and Menon, V. (2007). Temporal dynamics of basal ganglia response and connectivity during verbal working memory. Neuroimage 34, 1253-1269. doi: 10.1016/j.neuroimage.2006.08.056

Chen, S. H. A., and Desmond, J. E. (2005). Cerebrocerebellar networks during articulatory rehearsal and verbal working memory tasks. Neuroimage 24, 332-338. doi: 10.1016/j.neuroimage.2004.08.032

Cooper, F. E., Grube, M., Von Kriegstein, K., Kumar, S., English, P., Kelly, T. P., et al. (2012). Distinct critical cerebellar subregions for components of verbal working memory. Neuropsychologia 50, 189-197. doi: 10.1016/j.neuropsychologia.2011.11.017

Cowan, N. (2017). The many faces of working memory and short-term storage. Psychon Bull. Rev. 24, 1158-1170. doi: 10.3758/s13423-016-1191-6

Cowan, N., Rouder, J. N., Blume, C. L., and Saults, J. S. (2012). Models of verbal working memory capacity : what does it take. Psychol. Rev. 119, 480-499. doi: 10.1037/a0027791.Models

Crosson, B., Benefield, H., Cato, M. A., Sadek, J. R., Moore, A. B., Wierenga, C. E., et al. (2003). Left and right basal ganglia and frontal activity during language generation: contributions to lexical, semantic, and phonological processes. J. Int. Neuropsychol. Soc. 9, 1061-1177. doi: 10.1017/S135561770397010X

Crosson, B., Rao, S. M., Woodley, S. J., Rosen, A. C., Bobholz, J. A., Mayer, A., et al. (1999). Mapping of semantic, phonological, and orthographic verbal working memory in normal adults with functional magnetic resonance imaging. Neuropsychology 13, 171-187. doi: 10.1037/0894-4105.13.2.171

Daneman, A., and Carpenter, P. A. (1980). Individual differences in working memory and reading. J. Verbal Learn Verbal Behav. 19, 450-466. doi: 10.1016/S0022-5371(80)90312-6

Davis, S. W., Dennis, N. A., Daselaar, S. M., Fleck, M. S., and Cabeza, R. (2009). Qué PASA? The posterior-anterior shift in aging. Cereb. Cortex 18, 1201-1209. doi: 10.1093/cercor/bhm155.Qu

Deckersbach, T., Rauch, S. L., Buhlmann, U., Ostacher, M. J., Beucke, J. C., Nierenberg, A. A., et al. (2008). An fMRI investigation of working memory and sadness in females with bipolar disorder : a brief report. Bipolar Disord. 10, 928-942. doi: 10.1111/j.1399-5618.2008.00633.x

Desmond, J. E., Chen, S. H. A., DeRosa, E., Pryor, M. R., Pfefferbaum, A., and Sullivan, E. V. (2003). Increased frontocerebellar activation in alcoholics during verbal working memory: an fMRI study. Neuroimage 19, 1510-1520. doi: 10.1016/S1053-8119(03)00102-2

Desmond, J. E., Gabrieli, J. D. E., Wagner, A. D., Ginier, B. L., and Glover, G. H. (1997). Lobular patterns of cerebellar activation in verbal workingmemory and finger-tapping tasks as revealed by functional MRI. J. Neurosci. 17, 9675-9685. doi: 10.1523/JNEUROSCI.17-24-09675.1997

Dima, D., Jogia, J., and Frangou, S. (2014). Dynamic causal modeling of loaddependent modulation of effective connectivity within the verbal working memory network. Hum. Brain Mapp. 35, 3025-3035. doi: 10.1002/hbm.22382
Egger, M., Smith, G. D., Schneider, M., and Minder, C. (1997). Bias in meta-analysis detected by a simple, graphical test. BMJ. 315, 629-634. doi: 10.1136/bmj.315.7109.629

Engle, R. W., Laughlin, J. E., Tuholski, S. W., and Conway, A. R. A. (1999). Working memory, short-term memory, and general fluid intelligence: a latent-variable approach. J. Exp. Psychol. General. 128, 309-331. doi: 10.1037/0096-3445.128.3.309

Eriksson, J., Vogel, E. K., Lansner, A., Bergström, F., and Nyberg, L. (2015). Neurocognitive architecture of working memory. Neuron 88, 33-46. doi: 10.1016/j.neuron.2015.09.020

Forstmann, B. U., Jahfari, S., Scholte, H. S., Wolfensteller, U., van den Wildenberg, W. P. M., and Ridderinkhof, K. R. (2008). Function and structure of the right inferior frontal cortex predict individual differences in response inhibition: a model-based approach. J. Neurosci. 28, 9790-9796. doi: 10.1523/JNEUROSCI.1465-08.2008

Frank, M. J., Loughry, B., and O'Reilly, R. C. (2001). Interactions between frontal cortex and basal ganglia in working memory: a computational model. Cogn. Affect. Behav. Neurosci. 1, 137-160. doi: 10.3758/CABN.1.2.137

Fukuda, K., Vogel, E., Mayr, U., and Awh, E. (2010). Quantity, not quality: The relationship between fluid intelligence and working memory capacity. Psychonomic Bull. Rev. 17, 673-679. doi: 10.3758/17.5.673

Fullana, M. A., Albajes-Eizagirre, A., Soriano-Mas, C., Vervliet, B., Cardoner, N., Benet, O., et al. (2018). Fear extinction in the human brain: A meta-analysis of fMRI studies in healthy participants. Neurosci. Biobehav. Rev.. 88, 16-25. doi: 10.1016/j.neubiorev.2018.03.002

Garrett, A., Kelly, R., Gomez, R., Keller, J., Schatzberg, A. F., and Reiss, A. L. (2011). Aberrant brain activation during a working memory task in psychotic major depression. Am. J. Psychiatry 168, 173-182. doi: 10.1176/appi.ajp.2010.09121718

Gruber, O., Tost, H., Henseler, I., Schmael, C., Scherk, H., Ende, G., et al. (2010). Pathological amygdala activation during working memory performance: evidence for a pathophysiological trait marker in bipolar affective disorder. Hum. Brain Mapp. 31, 115-125. doi: 10.1002/hbm.20849

Hayter, A. L., Langdon, D. W., and Ramnani, N. (2007). Cerebellar contributions to working memory. Neuroimage 36, 943-954. doi: 10.1016/j.neuroimage.2007.03.011

Henson, R. N. A., Burgess, N., and Frith, C. D. (2000). Recoding, storage, rehersal, and grouping in verbal short-term memory: an fMRI study. Neuropsychologia 38, 426-440. doi: 10.1016/S0028-3932(99)00098-6

Higgins, J. P. T., and Thompson, S. G. (2002). Quantifying heterogeneity in a meta-analysis. Stat. Med.. 21, 1539-1558. doi: 10.1002/sim.1186

Hokkanen, L. S. K., Kauranen, V., Roine, R. O., Salonen, O., and Kotila, M. (2006). Subtle cognitive deficits after cerebellar infarcts. Eur. J. Neurol. 13, 161-170. doi: 10.1111/j.1468-1331.2006.01157.x

Höller-Wallscheid, M. S., Thier, P., Pomper, J. K., and Lindner, A. (2017). Bilateral recruitment of prefrontal cortex in working memory is associated with task demand but not with age. Proc. Natl. Acad. Sci. U.S.A. 114, 830-839. doi: 10.1073/pnas.1601983114

Honey, G. D., Bullmore, E. T., and Sharma, T. (2000). Prolonged reaction time to a verbal working memory task predicts increased power of posterior parietal cortical activation. Neuroimage 12, 495-503. doi: 10.1006/nimg.2000.0624

Inase, M., Tokuno, H., Nambu, A., Akazawa, T., and Takada, M. (1999). Corticostriatal and corticosubthalamic input zones from the presupplementary motor area in the macaque monkey: comparison with the input zones from the supplementary motor area. Brain Res.. 833, 191-201. doi: 10.1016/S0006-8993(99)01531-0

Japee, S., Holiday, K., Satyshur, M. D., Mukai, I., and Ungerleider, L. G. (2015). A role of right middle frontal gyrus in reorienting of attention: a case study. Front. Syst. Neurosci. 9:23. doi: 10.3389/fnsys.2015.00023

Johnson, M. R., Morris, N. A., Astur, R. S., Calhoun, V. D., Mathalon, D. H., Kiehl, K. A., et al. (2006). A functional magnetic resonance imaging study of working memory abnormalities in schizophrenia. Biol. Psychiatry 60, 11-21. doi: 10.1016/j.biopsych.2005.11.012

Joseph, J. E., Swearingen, J. E., Corbly, C. R., Curry, T. E., and Kelly, T. H. (2012). Influence of estradiol on functional brain organization for working memory. Neuroimage 59, 2923-2931. doi: 10.1016/j.neuroimage.2011. 09.067 
Just, M. A., and Carpenter, P. A. (1992). A capacity theory of comprehension: individual differences in working memory. Psychol. Rev. 99, 122-149. doi: 10.1037/0033-295X.99.1.122

Kane, M. J., and Engle, R. W. (2003). Working-memory capacity and the control of attention: the contributions of goal neglect, response competition, and task set to Stroop interference. J. Exp. Psychol. General 132, 47-70. doi: 10.1037/0096-3445.132.1.47

Karlsgodt, K. H., Shirinyan, D., Van Erp, T. G. M., Cohen, M. S., and Cannon, T. D. (2005). Hippocampal activations during encoding and retrieval in a verbal working memory paradigm. Neuroimage 25, 1224-1231. doi: 10.1016/j.neuroimage.2005.01.038

Kim, H. (2010). Dissociating the roles of the default-mode, dorsal, and ventral networks in episodic memory retrieval. Neuroimage 50, 1648-1657. doi: 10.1016/j.neuroimage.2010.01.051

Kirchner, W. K. (1958). Age differences in short-term retention of rapidly changing information. J. Exp. Psychol.. 55, 352-358. doi: 10.1037/h0043688

Kirschen, M. P., Chen, S. H. A., and Desmond, J. E. (2010). Modality specific cerebro-cerebellar activations in verbal working memory: an fMRI study. Behav. Neurol. 23, 51-63. doi: 10.3233/BEN-2010-0266

Kirschen, M. P., Chen, S. H. A., Schraedley-Desmond, P., and Desmond, J. E. (2005). Load- and practice-dependent increases in cerebro-cerebellar activation in verbal working memory: An fMRI study. Neuroimage. 24, 462-472. doi: 10.1016/j.neuroimage.2004.08.036

Knops, A., Nuerk, H. C., Fimm, B., Vohn, R., and Willmes, K. (2006). A special role for numbers in working memory? An fMRI study. Neuroimage 29, 1-14. doi: 10.1016/j.neuroimage.2005.07.009

Koelsch, S., Schulze, K., Sammler, D., Fritz, T., Müller, K., and Gruber, O. (2009). Functional architecture of verbal and tonal working memory: an fMRI study. Hum. Brain Mapp. 30, 859-873. doi: 10.1002/hbm.20550

Lejbak, L., Crossley, M., and Vrbancic, M. (2011). A male advantage for spatial and object but not verbal working memory using the n-back task. Brain Cogn. 76, 191-196. doi: 10.1016/j.bandc.2010.12.002

Lemaitre, H., Goldman, A. L., Sambataro, F., Verchinski, B. A., MeyerLindenberg, A., Weinberger, D. R., and Mattay, V. S. (2012). Normal agerelated brain morphometric changes: Nonuniformity across cortical thickness, surface area and gray matter volume? Neurobiol. Aging 33, 617.e1-617.e9. doi: 10.1016/j.neurobiolaging.2010.07.013

Lewis, S. J. G., Dove, A., Robbins, T. W., Barker, R. A., and Owen, A. M. (2004). Striatal contributions to working memory: a functional magnetic resonance imaging study in humans. Eur. J. Neurosci. 19, 755-760. doi: 10.1111/j.1460-9568.2003.03108.x

Lim, H.-K., Juh, R., Pae, C.-U., Lee, B.-T., Yoo, S.-S., Ryu, S.-H., et al. (2008). Altered verbal working memory process in patients with Alzheimer's disease. Neuropsychobiology 57, 181-187. doi: 10.1159/000147471

Lythe, K. E., Williams, S. C. R., Anderson, C., Libri, V., and Mehta, M. A. (2012). Frontal and parietal activity after sleep deprivation is dependent on task difficulty and can be predicted by the fMRI response after normal sleep. Behav. Brain Res. 233, 62-70. doi: 10.1016/j.bbr.2012.04.050

Makris, N., Kennedy, D. N., McInerney, S., Sorensen, A. G., Wang, R., Caviness, V. S., and Pandya, D. N. (2005). Segmentation of subcomponents within the superior longitudinal fascicle in humans: a quantitative, in vivo, DT-MRI study. Cereb. Cortex 15, 854-869. doi: 10.1093/cercor/bhh186

Makris, N., Papadimitriou, G. M., Kaiser, J. R., Sorg, S., Kennedy, D. N., and Pandya, D. N. (2009). Delineation of the middle longitudinal fascicle in humans: a quantitative, in vivo, DT-MRI study. Cereb. Cortex 19, 777-785. doi: 10.1093/cercor/bhn124

Marquand, A. F., Mourão-Miranda, J., Brammer, M. J., Cleare, A. J., and Fu, C. H. Y. (2008). Neuroanatomy of verbal working memory as a diagnostic biomarker for depression. Neuroreport 19, 1507-1511. doi: 10.1097/WNR.0b013e328310425e

Marvel, C. L., and Desmond, J. E. (2010). The contributions of cerebrocerebellar circuitry to executive verbal working memory. Cortex 46, 880-895. doi: 10.1016/j.cortex.2009.08.017

McMillan, K. M., Laird, A. R., Witt, S. T., and Meyerand, M. E. (2007). Self-paced working memory: Validation of verbal variations of the n-back paradigm. Brain Res. 1139, 133-142. doi: 10.1016/j.brainres.2006.12.058

McNab, F., Leroux, G., Strand, F., Thorell, L., Bergman, S., and Klingberg, T. (2008). Common and unique components of inhibition and working memory: an fMRI, within-subjects investigation. Neuropsychologia 46, 2668-2682. doi: 10.1016/j.neuropsychologia.2008.04.023

Meisenzahl, E. M., Scheuerecker, J., Zipse, M., Ufer, S., Wiesmann, M., Frodl, T., et al. (2006). Effects of treatment with the atypical neuroleptic quetiapine on working memory function: a functional MRI follow-up investigation. Eur. Arch. Psychiatry Clin. Neurosci. 256, 522-531. doi: 10.1007/s00406-006-0687-x

Meule, A. (2017). Reporting and interpreting working memory performance in n-back tasks. Front. Psychol. 8:352. doi: 10.3389/fpsyg.2017.00352

Mink, J. W. (1996). The basal ganglia: focused selection and inhibition of competing motor programs. Progr. Neurobiol. 50, 381-425. doi: 10.1016/S0301-0082(96)00042-1

Monks, P. J., Bullmore, E. T., Suckling, J., Brammer, M. J., Williams, S. C., Simmons, A., et al. (2004). A functional MRI study of working memory task in euthymic bipolar disorder: evidence for task-specific dysfunction. Bipolar Disord. 6, 550-564. doi: 10.1111/j.1399-5618.2004.00147.x

Moore, A. B., Li, Z., Tyner, C. E., Hu, X., and Crosson, B. (2013). Bilateral basal ganglia activity in verbal working memory. Brain Lang. 125, 316-323. doi: 10.1016/j.bandl.2012.05.003

Mordecai, K. L., Rubin, L. H., and Maki, P. M. (2008). Effects of menstrual cycle phase and oral contraceptive use on verbal memory. Horm. Behav. 54, 286-293. doi: 10.1016/j.yhbeh.2008.03.006

Mu, Q., Nahas, Z., Johnson, K. A., Yamanaka, K., Mishory, A., Koola, J., et al. (2005). Decreased cortical response to verbal working memory following sleep deprivation. Sleep 28, 55-67. doi: 10.1093/sleep/28.1.55

Müller, V. I., Cieslik, E. C., Laird, A. R., Fox, P. T., Radua, J., Mataix-Cols, D., et al. (2018). Ten simple rules for neuroimaging meta-analysis. Neurosci. Biobehav. Rev. 84, 151-161. doi: 10.1016/j.neubiorev.2017.11.012

Narayanan, N. S., Prabhakaran, V., Bunge, S. A., Christoff, K., Fine, E. M., and Gabrieli, J. D. E. (2005). The role of the prefrontal cortex in the maintenance of verbal working memory: an event-related fMRI analysis. Neuropsychology 19, 223-232. doi: 10.1037/0894-4105.19.2.223

Nee, D. E., Brown, J. W., Askren, M. K., Berman, M. G., Demiralp, E., Krawitz, A., and Jonides, J. (2013). A meta-Analysis of executive components of working memory. Cereb Cortex 23, 264-282. doi: 10.1093/cercor/bhs007

Ng, H. B. T., Kao, K. L. C., Chan, Y. C., Chew, E., Chuang, K. H., and Chen, S. H. A. (2016). Modality specificity in the cerebro-cerebellar neurocircuitry during working memory. Behav. Brain Res. 305, 164-173. doi: 10.1016/j.bbr.2016.02.027

Norbury, R., Godlewska, B., and Cowen, P. J. (2014). When less is more: a functional magnetic resonance imaging study of verbal working memory in remitted depressed patients. Psychol. Med. 44, 1197-1203. doi: $10.1017 /$ S0033291713001682

Oberauer, K. (2010). Design for a working memory. Psychol. Learn. Motivat. 51, 45-100. doi: 10.1016/S0079-7421(09)51002-X

Oberauer, K., and Hein, L. (2012). Attention to information in working memory. Curr. Dir. Psychol. Sci. 21, 164-169. doi: 10.1177/0963721412444727

Owen, A. M., McMillan, K. M., Laird, A. R., and Bullmore, E. (2005). Nback working memory paradigm: a meta-analysis of normative functional neuroimaging studies. Hum. Brain Mapp. 25, 46-59. doi: 10.1002/hbm.20131

Park, D. C., and Reuter-Lorenz, P. (2009). The adaptive brain : aging and neurocognitive scaffolding. Annu. Rev. Neurosci. 60, 173-196. doi: 10.1146/annurev.psych.59.103006.093656

Paule, M. G., Bushnell, P. J., Maurissen, J. P. J., Wenger, G. R., Buccafusco, J. J., Chelonis, J. J., et al. (1998). Symposium overview : the use of delayed matchingto-sample procedures in studies of short-term memory in animals and humans. Neurotoxicol. Teratol. 20, 493-502. doi: 10.1016/S0892-0362(98)00013-0

Paulesu, E., Frith, C. D., and Frackowiak, R. S. (1993). The neural correlates of the verbal component of working memory. Nature 362, 342-345. doi: $10.1038 / 362342 \mathrm{a} 0$

Petrides, M., Alivisatos, B., Meyer, E., and Evans, A. C. (1993). Functional activation of the human frontal cortex during the performance of verbal working memory tasks. Neurobiology 90, 878-882. doi: 10.1073/pnas.90. 3.878

Pleger, B., and Timmann, D. (2018). The role of the human cerebellum in linguistic prediction, word generation and verbal working memory: evidence from brain imaging, non-invasive cerebellar stimulation and lesion studies. Neuropsychologia 115, 204-210. doi: 10.1016/j.neuropsychologia.2018. 03.012 
Radua, J., and Mataix-Cols, D. (2009). Voxel-wise meta-analysis of grey matter changes in obsessive-compulsive disorder. Br. J. Psychiatry 195, 393-402. doi: 10.1192/bjp.bp.108.055046

Radua, J., Rubia, K., Canales-Rodríguez, E. J., Pomarol-Clotet, E., Fusar-Poli, P., and Mataix-Cols, D. (2014). Anisotropic kernels for coordinatebased meta-analyses of neuroimaging studies. Front. Psychiatry 5:13. doi: 10.3389/fpsyt.2014.00013

Ragland, J. D., Turetsky, B. I., Gur, R. C., Gunning-Dixon, F., Turner, T., Schroeder, L., et al. (2002). Working memory for complex figures: an fMRI comparison of letter and fractal n-back tasks. Neuropsychology 16, 370-379. doi: 10.1037/0894-4105.16.3.370

Ravizza, S. M., Delgado, M. R., Chein, J. M., Becker, J. T., and Fiez, J. A. (2004). Functional dissociations within the inferior parietal cortex in verbal working memory. Neuroimage 22, 562-573. doi: 10.1016/j.neuroimage.2004.01.039

Ravizza, S. M., McCormick, C. A., Schlerf, J. E., Justus, T., Ivry, R. B., and Fiez, J. A. (2006). Cerebellar damage produces selective deficits in verbal working memory. Brain 129, 306-320. doi: 10.1093/brain/awh685

Raz, N., Lindenberger, U., Rodrigue, K. M., Kennedy, K. M., Head, D., Williamson, A., et al. (2005). Regional brain changes in aging healthy adults: general trends, individual differences and modifiers. Cereb. Cortex 15, 1676-1689. doi: $10.1093 /$ cercor/bhi044

Reuter-Lorenz, P. A., and Cappell, K. A. (2008). Neurocognitive aging and the compensation hypothesis. Curr. Dir. Psychol. Sci. 17, 177-182. doi: $10.1111 / j .1467-8721.2008 .00570 . x$

Reuter-Lorenz, P. A., Jonides, J., Smith, E. E., Hartley, A., Miller, A., Marshuetz, C., and Koeppe, R. A. (2000). Age differences in the frontal lateralization of verbal and spatial working memory revealed by PET. J. Cogn. Neurosci. 12, 174-187. doi: $10.1162 / 089892900561814$

Reuter-lorenz, P. A., Stanczak, L., and Miller, A. C. (1999). Neural recruitment and cognitive aging : two hemispheres are better than one, especially as you age. Psychol. Sci. 10, 494-500. doi: 10.1111/1467-9280.00195

Rey-Mermet, A., and Gade, M., Souza, A. S., von Bastian, C. C., and Oberauer, K. (2019). Is executive control related to working memory capacity and fluid intelligence? J. Exp. Psychol. General. doi: 10.1037/xge0000593

Rieckmann, A., Pudas, S., and Nyberg, L. (2017). Longitudinal changes in component processes of working memory. Eneuro 4, 1-9. doi: 10.1523/ENEURO.0052-17.2017

Rottschy, C., Langner, R., Dogan, I., Reetz, K., Laird, A. R., Schulz, J. B., et al. (2012). Modelling neural correlates of working memory: a coordinate-based meta-analysis. Neuroimage 60, 830-846. doi: 10.1016/j.neuroimage.2011.11.050

Scheuerecker, J., Ufer, S., Zipse, M., Frodl, T., Koutsouleris, N., Zetzsche, T., et al. (2008). Cerebral changes and cognitive dysfunctions in medicationfree schizophrenia - An fMRI study. J. Psychiatr. Res. 42, 469-476. doi: 10.1016/j.jpsychires.2007.04.001

Schlösser, R. G. M., Koch, K., Wagner, G., Nenadic, I., Roebel, M., Schachtzabel, C., et al. (2008). Inefficient executive cognitive control in schizophrenia is preceded by altered functional activation during information encoding: an fMRI study. Neuropsychologia 46, 336-347. doi: 10.1016/j.neuropsychologia.2007.07.006

Schmahmann, J. (1991). An emerging concept: the cerebellar contribution to higher function. Arch. Neurol. 48, 1178-1187. doi: 10.1001/archneur.1991.00530230086029

Schmahmann, J. D. (1996). From movement to thought: anatomic substrates of the cerebellar contribution to cognitive processing. Hum. Brain Mapp. 4, 174-198. doi: 10.1002/(SICI) 1097-0193(1996)4:3<174::AID-HBM3>3.0.CO;2-0

Schmidt, H., Jogia, J., Fast, K., Christodoulou, T., Haldane, M., Kumari, V., and Frangou, S. (2009). No gender differences in brain activation during the N-back task: an fMRI study in healthy individuals. Hum. Brain Mapp. 30, 3609-3615. doi: $10.1002 / \mathrm{hbm} .20783$

Seghier, M. L. (2013). The angular gyrus: multiple functions and multiple subdivisions. Neuroscientist 19, 43-61. doi: 10.1177/1073858412440596

Seo, J., Kim, S.-H., Kim, Y.-T., Song, H., Lee, J., Kim, S.-H., et al. (2012). Working memory impairment in fibromyalgia patients associated with altered frontoparietal memory network. PLoS ONE 7:e37808. doi: 10.1371/journal.pone.0037808

Silveri, M. C., Di Betta, A. M., Filippini, V., Leggio, M. G., and Molinari, M. (1998). Verbal short-term store-rehearsal system and the cerebellum. Evidence from a patient with a right cerebellar lesion. Brain 121, 2175-2187. doi: 10.1093/brain/121.11.2175
Smith, E. E., and Jonides, J. (1998). Neuroimaging analyses of human working memory. Psychol. Neurobiol. 95, 12061-12068. doi: 10.1073/pnas.95.20. 12061

Spaniol, J., Davidson, P. S. R., Kim, A. S. N., Han, H., Moscovitch, M., and Grady, C. L. (2009). Event-related fMRI studies of episodic encoding and retrieval: meta-analyses using activation likelihood estimation. Neuropsychologia 47, 1765-1779. doi: 10.1016/j.neuropsychologia.2009.02.028

Sternberg, S. (1966). High-speed scanning in human memory. Science 153, 652-654. doi: 10.1126/science.153.3736.652

Stoodley, C. J., and Schmahmann, J. D. (2009). Functional topography in the human cerebellum: a meta-analysis of neuroimaging studies. Neuroimage 44, 489-501. doi: 10.1016/j.neuroimage.2008.08.039

Stroup, D. F., Berlin, J. A., Morton, S. C., Olkin, I., Williamson, G. D., Rennie, D., et al. (2000). Meta-analysis of observational studies in epidemiology. JAMA. 283, 2008-2012. doi: 10.1001/jama.283.15.2008

Thürling, M., Hautzel, H., Küper, M., Stefanescu, M. R., Maderwald, S., Ladd, M. E., and Timmann, D. (2012). Involvement of the cerebellar cortex and nuclei in verbal and visuospatial working memory: a 7T fMRI study. Neuroimage 62, 1537-1550. doi: 10.1016/j.neuroimage.2012.05.037

Tomlinson, S. P., Davis, N. J., Morgan, H. M., and Bracewell, R. M. (2014), Cerebellar contributions to verbal working memory. Cerebellum 13, 354-361. doi: $10.1007 / \mathrm{s} 12311-013-0542-3$

Tsapkini, K., and Rapp, B. (2010). The orthography-specific functions of the left fusiform gyrus: evidence of modality and category specificity. Cortex 46, 185-205. doi: 10.1016/j.cortex.2009.02.025

Tzourio-Mazoyer, N., Landeau, B., Papathanassiou, D., Crivello, F., Etard, O., Delcroix, N., et al. (2002). Automated anatomical labeling of activations in SPM using a macroscopic anatomical parcellation of the MNI MRI single-subject brain. Neuroimage 15, 273-289. doi: 10.1006/nimg.2001.0978

Uddin, L. Q., Supekar, K., Amin, H., Rykhlevskaia, E., Nguyen, D. A., Greicius, M. D., and Menon, V. (2010). Dissociable connectivity within human angular gyrus and intraparietal sulcus: Evidence from functional and structural connectivity. Cereb. Cortex 20, 2636-2646. doi: 10.1093/cercor/ bhq011

Unsworth, N., and Engle, R. W. (2007). The nature of individual differences in working memory capacity: active maintenance in primary memory and controlled search from secondary memory. Psychol. Rev. 114, 104-132. doi: 10.1037/0033-295X.114.1.104

Valera, E. M., Faraone, S. V., Biederman, J., Poldrack, R. A., and Seidman, L. J. (2005). Functional neuroanatomy of working memory in adults with attention-deficit/hyperactivity disorder. Biol. Psychiatry 57, 439-447. doi: 10.1016/j.biopsych.2004.11.034

Veltman, D. J., Rombouts, S. A. R. B., and Dolan, R. J. (2003). Maintenance versus manipulation in verbal working memory revisited: an fMRI study. Neuroimage 18, 247-256. doi: 10.1016/S1053-8119(02)00049-6

Wager, T. D., and Smith, E. E. (2003). Neuroimaging studies of working memory: a meta-analysis. Cogn. Affect. Behav. Neurosci. 3, 255-274. doi: 10.3758/CABN.3.4.255

Wager, T. D., Sylvester, C. Y. C., Lacey, S. C., Nee, D. E., Franklin, M., and Jonides, J. (2005). Common and unique components of response inhibition revealed by fMRI. Neuroimage 27, 323-340. doi: 10.1016/j.neuroimage.2005.01.054

Walter, H., Bretschneider, V., Grön, G., Zurowski, B., Wunderlich, A. P., Tomczak, R., et al. (2003). Evidence for quantitative domain dominance for verbal and spatial working memory in frontal and parietal cortex. Cortex 39, 897-911. doi: 10.1016/S0010-9452(08)70869-4

Walter, H., Wolf, R. C., Spitzer, M., and Vasic, N. (2007). Increased left prefrontal activation in patients with unipolar depression: an event-related, parametric, performance-controlled fMRI study. J. Affect. Disord. 101, 175-185. doi: 10.1016/j.jad.2006.11.017

Wiley, J., and Jarosz, A. F. (2012). Working memory capacity, attentional focus, and problem solving. Curr. Dir. Psychol. Sci. 21, 258-262. doi: $10.1177 / 0963721412447622$

Wishart, H. A., Saykin, A. J., Rabin, L. A., Santulli, R. B., Flashman, L. A., Guerin, S. J., et al. (2006). Increased brain activation during working memory in cognitively intact adults with the APOE $\varepsilon 4$ allele. Am. J. Psychiatry 163, 1603-1610. doi: 10.1176/ajp.2006.163.9.1603

Wolf, R. C., Vasic, N., and Walter, H. (2006). Differential activation of ventrolateral prefrontal cortex during working memory retrieval. 
Neuropsychologia 44, 2558-2563. doi: 10.1016/j.neuropsychologia.2006. 05.015

Wu, C., Zheng, Y., Li, J., Zhang, B., Li, R., Wu, H., et al. (2017). Activation and functional connectivity of the left inferior temporal gyrus during visual speech priming in healthy listeners and listeners with schizophrenia. Front. Neurosci. 11:107. doi: 10.3389/fnins.2017.00107

Yan, X., Zhang, J., Gong, Q., and Weng, X. (2011). Prolonged high-altitude residence impacts verbal working memory: an fMRI study. Exp. Brain Res. 208, 437-445. doi: 10.1007/s00221-010-2494-x

Yoo, S. S., Paralkar, G., and Panych, L. P. (2004). Neural substrates associated with the concurrent performance of dual working memory tasks. Int. J. Neurosci. 114, 613-631. doi: 10.1080/00207450490430561

Zilles, D., Lewandowski, M., Vieker, H., Henseler, I., Diekhof, E., Melcher, T., et al. (2016). Gender differences in verbal and visuospatial working memory performance and networks. Neuropsychobiology 73, 52-63. doi: $10.1159 / 000443174$

Conflict of Interest Statement: The authors declare that the research was conducted in the absence of any commercial or financial relationships that could be construed as a potential conflict of interest.

Copyright (c) 2019 Emch, von Bastian and Koch. This is an open-access article distributed under the terms of the Creative Commons Attribution License (CC BY).

The use, distribution or reproduction in other forums is permitted, provided the original author(s) and the copyright owner(s) are credited and that the original publication in this journal is cited, in accordance with accepted academic practice. No use, distribution or reproduction is permitted which does not comply with these terms. 